Erschienen in: Harras, Gisela/Proost, Kristel/Winkler, Edeltraud: Handbuch deutscher Kommunikationsverben. Teil 2: Lexikalische Strukturen. - Berlin/New York: de Gruyter, 2007. S. 367-397.

(Schriften des Instituts für deutsche Sprache 10.2)

\title{
Kristel Proost
}

\section{Gegensatzrelationen von Sprechaktverben}

Sprechaktverben stehen, wie auch andere Einheiten des Lexikons, in unterschiedlichen Arten von Gegensarzbeziehungen zueinander. Das Spektrum der Gegensatzrelationen umfasst eine Vielfalt unterschiedlicher Phänomene wie Komplementarität, Kontrarität, direktionale Opposition, Subkontrarität, Konversivität, Reversivität und Dualität (vgl. Cruse 1986, Lang 1995, Cruse et al. 2002), die alle negationsinvolvierend, aber für den Wortschatzausschnitt der Sprechaktverben nicht alle gleichermaßen relevant sind. Gegensarzrelationen von Wörtern sind meist bei Adjektiven, seltener auch bei Adverbien, Nomen und Verben untersucht worden. Im Unterschied zu den klassischen Beispielen antonymischer Verben wie z. B. lieben-hassen, kommen-gehen und kaufenverkaufen lassen sich Sprechaktverben nur schwer in das Spektrum der Gegensatzrelationen einordnen. Im Folgenden werden die Kriterien, die zur Bestimmung unterschiedlicher Typen von Gegensatzrelationen angeführt worden sind, diskutiert und auf den Wortschatzbereich der Sprechaktverben angewendet.

\section{Typen von Gegensatzrelationen}

\subsection{Komplementarität (Antonymie, kontradiktorisch)}

Komplementarität ist eine Relation des Bedeutungsgegensatzes zwischen zwei lexikalischen Einheiten $\mathrm{L}(\mathrm{a})$ und $\mathrm{L}(\mathrm{b})$, die eine bestimmte konzeptuelle Domäne in zwei sich gegenseitig ausschlieBende Bereiche einteilen: wenn das durch den einen Ausdruck lexikalisierte Konzept (a oder b) nicht zum einen Bereich gehört, gehört es automatisch zum anderen (vgl. Cruse 1986, 198f.). Wenn zwei lexikalische Ausdrücke komplementäre (oder kontradiktorische) Antonyme sind, gelten die folgenden Implikationsbeziehungen:

(1) $a \rightarrow \neg b \& \neg a \rightarrow b$

Wenn $\mathbf{x}$ ein $\mathrm{a}$ ist, ist es nicht ein $\mathrm{b}$, und wenn $\mathbf{x}$ nicht ein $\mathbf{a}$ ist, ist es ein $\mathrm{b}$. Das heißt, dass $\mathrm{a}$ und $\mathrm{b}$ nicht beide zugleich zutreffen und nicht beide zugleich nicht zutreffen können (vgl. Lang 1995. 32; Lehrer 2002, 503). Beispiele komplementärer Adjektivpaare sind gerade-ungerade, tot-lebend(ig), ledig-verheiratet und möglich-unmöglich. Nach Löbner ist Komplementarität häufiger bei Nomen anzutreffen. Beispiele sind Wortpaare, die sich durch gegensärzliche Geschlechtsspezifikation unterscheiden wie Frau-Mann, Arztin-Arzt, Abgeordnete-Abgeordneter oder Paare wie Mitglied-Nichtmitglied und Inland-Ausland (vgl. Löbner 2003, 127). Lang erwähnt schlafen-wach sein als Beispiel eines Paares komplementärer Verben (vgl. Lang 1995, 32).

Lang weist darauf hin, dass Komplementarität als lexikalische Ordnungsrelation eher schwach ausgeprägt zu sein scheint. Echte Beispiele seien entweder in der Fachlexik anzutreffen (organischanorganisch, metallisch-nichtmetallisch) oder sie zeigten deutliche Abweichungen von der Kategorienkonstanz (tot sein-leben, schlafen-wach sein) (vgl. Lang 1995, 32 f.). Komplementäre Verben kommen im Wortschatzausschnitt der Sprechaktverben nicht vor. Als Beispiel eines komplementären Verbpaares kommt allenfalls reden-schweigen in Frage, das aber nicht aus Sprechaktverben besteht: reden ist ein Kommunikationsverb, d. h., ein Verb, mit dem im Unterschied zu Sprechaktverben im eigentlichen Sinn keine Sprechereinstellungen wie etwa eine bestimmte pro- 
positionale Einstellung oder eine bestimmte Sprecherabsicht lexikalisiert sind; schweigen ist weder ein Sprechakt- noch ein Kommunikationsverb.

\subsection{Kontrarität (Antonymie, konträr)}

Kontrarität ist eine Relation des Bedeutungsgegensatzes zwischen zwei lexikalischen Ausdrücken L(a) und L(b), die entgegengesetzte Extreme auf einer Skala von Möglichkeiten bezeichnen (vgl. Löbner 2003, 123). Typische Beispiele konträrer Antonyme sind Adjektivpaare wie groß-klein, dick-dünn, alt-jung, glücklich-unglücklich, gut-schlecht, kalt-heiß und kühl-warm. Die Skala der Möglichkeiten, die durch konträre Antonyme bezeichnet werden, ist nach oben und nach unten offen und enthält einen neutralen Mittelbereich, der weder vom einen noch vom anderen Ausdruck eines konträren Antonymenpaares bezeichnet wird. Dementsprechend teilen konträre Antonyme eine konzeptuelle Domäne nicht in zwei sich gegenseitig ausschließende Bereiche ein: konträre Antonyme sind nicht komplementär (vgl. Cruse 1986, 204; Löbner 2003, 124), was sich in den Implikationsverhältnissen zeigt, die zwischen konträren Antonymen gelten:

(2) $\mathrm{a} \rightarrow \neg \mathrm{b} \& \mathrm{~b} \rightarrow \neg \mathrm{a}$

Wenn $x$ ein $a$ ist, ist es nicht ein $b$, und wenn $x$ ein $b$ ist, ist es nicht ein $a$; $a$ und $b$ können nicht beide zugleich zutreffen, aber beide können zugleich nicht zutreffen (vgl. Lang 1995, 33). X ist nicht kurz ist beispielsweise nicht äquivalent zu $X$ ist lang, weil etwas sowohl ,nicht kur' als auch ,nicht lang' sein kann. $X$ ist kurz impliziert $X$ ist nicht lang und $X$ ist lang impliziert $X$ ist nicht kurz, aber $X$ ist nicht kurz impliziert nicht $X$ ist lang und $X$ ist nicht lang impliziert nicht $X$ ist kurz. Bezeichnungen für den mittleren Bereich sind selten (vgl. Cruse 1986, 205; Lehrer 2002, 501; Löbner 2003, 124). Ausnahmen sind lauwarm, halbdunkel und mittelschlank. Konträre Antonyme sind meist Adjektive, aber es gibt auch Paare konträrer Nomen (Liebe-Hass, Stille-Lärm), Verben (lieben-hassen, ermutigen-entmutigen), Pronomen (alles-nichts) und Adverbien (immer-nie, oft-selten) (vgl. Löbner 2003, 125).

Langs Definition von konträrer Antonymie (vgl. Lang 1995, 33) nimmt nur auf die Implikationsverhältnisse Bezug, die zwischen konträren Antonymen gelten. Damit ist unklar, ob er konträre Antonymie als eine Eigenschaft graduierbarer Ausdrücke ansieht. Die Tatsache, dass er kommen und gehen als Beispiele konträrer Antonyme anführt, lässt jedenfalls darauf schließen, dass konträre Antonymie nach Lang nicht auf skalare Ausdrücke beschränkt ist.

\subsection{Direktionale Opposition}

Direktionale Opposition wird von manchen Autoren als eine eigenständige Relation des Bedeutungsgegensatzes betrachtet (vgl. Lyons 1977, 281-287; Cruse 1986, 223-243; Löbner 2003, $125 \mathrm{f}$.). Mit direktionalen Gegensatzbezeichnungen wird auf entgegengeserzte Richtungen auf einer Raum- oder Zeitachse Bezug genommen (vgl. Löbner 2003, 127). Beispiele direktionaler Gegensatzbezeichnungen sind vorne-hinten, oben-unten, hoch-niedrig, steigen-fallen, heben-senken, kommen-gehen, vor-nach, gestern-morgen und vorhin-nachher.

Lang erwähnt hoch-niedrig, steigen-fallen, kommen-gehen und über-unter als Beispiele konträrer Antonyme und behandelt direktionale Opposition damit als einen Fall von konträrer Antonymie (vgl. Lang 1995, 33). Nach Löbner haben direktionale Gegensatzbezeichnungen viel mit konträren Antonymen gemeinsam. Für jedes Paar direktionaler Gegensatzbezeichnungen, die sich auf eine räumliche Achse beziehen, gebe es einen Bezugspunkt, von dem aus man entlang einer 
Raumachse in entgegengesetzte Richtungen blicken kann; dieser Bezugspunkt entspreche dem neutralen Bereich auf einer Skala konträrer Antonyme. Wie diese sei auch eine Raumachse in beide Richtungen unbegrenzt (vgl. Löbner 2003, 125). Auch Lyons betont, dass direktionale Opposition nicht immer von den anderen Typen von Gegensatzrelationen (bei Lyons konträre Antonymie, Komplementarität und Konversivität) unterschieden werden kann (vgl. Lyons 1977, 281). Ein Beispiel ist das Paar kaufen-verkaufen, dessen Elemente einerseits als direktionale Gegensätze (,in Besitz bringen“ vs. ,weggeben'), andererseits aber auch als Konverse betrachtet werden können (vgl. Löbner 2003, 126). Dies gilt auch für über-unter, vor-nach, oberhalb-unterhalb usw. Da Direktionalität auf einer Raum- oder Zeitachse für die Bedeutung von Sprechaktverben irrelevant ist, spielt direktionale Opposition für den Wortschatzausschnitt dieser Verben keine Rolle.

Cruse erwähnt Reversivität als eine besondere Art der direktionalen Opposition (vgl. Cruse 1986, 226-231). Unter "Reversivität" versteht Cruse eine Relation des Bedeutungsgegensatzes zwischen zwei Verben X und Y, die beide ein Ereignis bezeichnen, in dem ein bestimmtes Element $\mathrm{A}$ von einem Anfangszustand I (,initial state") zu einem Endzustand F (,final state") wechselt. Abhängig von der Bedeutung von $\mathrm{X}$ und $\mathrm{Y}$ können I und $\mathrm{F}$ bestimmte („determinate") oder auch unbestimmte („indeterminate“) Zustände sein (vgl. Cruse 1979, 959). Cruse erwähnt enter und leave als Beispiele von Reversiven, die mit Bezug auf einen Wechsel zwischen bestimmten Zuständen verwendet werden: In $A$ entered $B$ bezeichnet enter einen Wechsel von ,A nicht in $B^{\prime}$ (I) zu , $A$ in $B^{\prime}$ (F); in $A$ left B bezeichnet leave einen Wechsel von, A in B' (I) zu, A nicht in B' (F). Beispiele von Reversiven, mit denen auf einen Wechsel zwischen unbestimmten Zuständen Bezug genommen wird, sind lengthen und shorten: lengthen bezeichnet den Wechsel ,F länger als I" und shorten den Wechsel ,I länger als $F^{\prime}$. lengthen und shorten bezeichnen einen Wechsel zwischen unbestimmten Zuständen, weil die Prozesse des Länger-Machens und des Kürzens bei jeder beliebigen Länge anfangen und enden können (vgl. Cruse 1979, 959).

Cruse unterscheidet zwei Typen von Reversiven: unabhängige (,independent') und abhängige (,dependent") Reversive; Letztere nennt er "Restiturive'. Unter "unabhängigen Reversiven" versteht Cruse "reversive pairs whose members are logically independent of one another" (Cruse 1979, 960). Lengthen und shorten sind unabhängige Reversive: eine Äußerung wie Mary lengthened her dress impliziert nicht, dass Mary das Kleid vorher gekürzt hat, und Mary shortened her dress impliziert nicht, dass Mary das Kleid vorher länger gemacht hat (vgl. Cruse 1979, 960). Restitutive sind Paare von Reversiven, die "voneinander abhängig“ sind (Cruse 1979, 960). Cruses Beispiel ist remove-replace. der durch replace bezeichnete Zustandswechsel ist logisch abhängig von dem, der durch remove bezeichnet wird: etwas kann nur dann wieder an seinen Platz hingestellt werden, wenn es vorher von dort entfernt worden ist. Weitere Beispiele restitutiver Verbpaare sind nach Cruse stop-resume, leave-return, damage-repair und fall ill-recover (vgl. Cruse 2002, 509). Direktionale Opposition ist für den Wortschatzbereich der Sprechaktverben nicht relevant.

\subsection{Subkontrarität}

Die Relation der Subkontrarität ist als Ausprägung einer antonymischen Relation zu betrachten, weil sie negationsinvolvierend ist (vgl. Lang 1995, 33). Lang definiert Subkontrarität folgendermaßen:

(3) $\neg \mathrm{a} \rightarrow \mathrm{b} \& \neg \mathrm{b} \rightarrow \mathrm{a}$ 
Wenn $x$ nicht ein $a$ ist, ist es ein $b$, und wenn $x$ nicht ein $b$ ist, ist es ein $a$; $a$ und $b$ können einzeln oder beide zutreffen, aber nicht beide nicht. Nach Lang ist der durch Subkontrarität definierte Kontrast zwischen a und $b$ nur ein mittelbarer, weil a und b komparibel sind. Entsprechend schwach falle die semantische Differenz zwischen Belegen für $\mathrm{L}(\mathrm{a})$ und $\mathrm{L}(\mathrm{b})$ aus: möglich-unnötig, ungerade-teilbar, erlauben-freistellen usw. Semantische Subkontrarität stelle sich normalerweise erst in entsprechenden strukturellen Kontexten ein wie z. B. in Frauen oder Behinderte als Bewerber werden bevorzugt (vgl. Lang 1995, 33). Im Folgenden werden nur Relationen des Bedeutungsgegensatzes zwischen zwei lexikalischen Einheiten L(a) und L(b) diskutiert, die auch inkompatibel sind, d.h., deren Denotationen sich nicht überschneiden. Fälle von Subkontrarität werden daher nicht in Betracht gezogen.

\subsection{Konversivität}

Konversivität ist eine Relation des Bedeutungsgegensatzes, die auf einer unterschiedlichen Betrachtungsweise ein und desselben Verhältnisses beruht. Wenn eine Person X einer Person Y beispielsweise etwas gibt, kann diese Handlung aus der Sicht von $\mathrm{X}$ mit geben und aus der Sicht von Y mit nehmen bezeichnet werden (vgl. Roelcke 2002, 511 ). Nur mehrstellige Prädikatsausdrücke können in einer Beziehung der Konversivität zueinander stehen. Zwei mehrstellige Prädikatsausdrücke L(a) und L(b) sind genau dann zueinander konvers, wenn sie dieselbe Beziehung mit vertauschten Rollen ausdrücken (vgl. Löbner 2003, 128). Beispiele von Paaren konverser Ausdrücke sind kaufen-verkaufen, geben-erhalten, Nachfolger-Vorgänger, Elternteil-Kind, LehrerSchüler, nach-vor, über-unter usw.

Dass konverse Ausdrücke dieselbe Beziehung mit vertauschten Rollen ausdrücken, zeigt sich in der Äquivalenz von Sätzen, in denen die betreffenden Ausdrücke und ihre Argumente ausgetauscht werden (vgl. Cruse 1986, 231):

(4) $\mathrm{x}$ kauft ein Buch von $\mathrm{y} \leftrightarrow \mathrm{y}$ verkauft $\mathrm{x}$ ein Buch

(5) $\mathrm{x}$ ist der Nachfolger von $\mathrm{y} \leftrightarrow \mathrm{y}$ ist der Vorgänger von $\mathrm{x}$

(6) $\mathrm{x}$ ist über $\mathrm{y} \leftrightarrow \mathrm{y}$ ist unter $\mathrm{x}$

Verallgemeinernd kann die Form dieser äquivalenten Sätze folgendermaßen dargestellt werden:

(7) $a(x, y, \ldots) \leftrightarrow b(y, x, \ldots)$

Wenn $x$ ein a zu $y$ ist, ist $y$ ein b zu $x$ (vgl. Lang 1995, 34).

Dass der Kontrast zwischen zwei Konversen L(a) und L(b) negationsinvolvierend ist, zeigt sich nach Lang im Implikationsverhältnis in (8):

(8) $a(x, y, \ldots) \rightarrow \neg b(x, y, \ldots)$

Wenn $x$ ein a zu $y$ ist, ist es nicht ein b zu $y$.

Löbner weist darauf hin, dass man den Begriff der Konversivität auf nicht-reziproke Ausdrücke beschränken muss, wenn man ihn als echte Gegensatzrelation definieren möchte. Reziproke oder selbst-konverse Ausdrücke wie etwa gleich, verschieden und Nachbar bezeichnen symmetrische Beziehungen: Wenn $\mathrm{x}$ und $\mathrm{y}$ gleich oder verschieden sind, sind es auch $\mathrm{y}$ und $\mathrm{x}$; wenn $\mathrm{x}$ ein Nachbar von $y$ ist, ist y auch ein Nachbar von x usw. (vgl. Löbner 2003, 129). 
Als Beispiel eines Paares konverser kommunikativer Ausdrücke kommt fragen-antworten in Frage, das aus einem Sprechaktverb (fragen) und einem gesprächs- und themenstrukturierenden Verb (antworten) besteht. fragen und antworten stehen nach Lyons "permutationell" in der gleichen Beziehung wie Konverse ( $x$ fragt $y$ etwas; $y$ antwortet $x$ etwas). Während Sätze, in denen "echte“ Konverse wie kaufen und verkaufen sowie ihre Argumente vertauscht werden, sich gegenseitig implizieren, sei dies bei Sätzen mit fragen und antworten allerdings nicht der Fall: $x$ kauft $z$ von $y$ impliziert $y$ verkauft $x z$ und umgekehrt, aber $x$ fragt $y$ etwas impliziert nicht $y$ antwortet $x$ etwas. Zwischen den Akten, auf die mit fragen bzw. antworten Bezug genommen wird, bestehe vielmehr eine Relation der zeitlichen Abfolge: auf einen Akt des Fragens wird eine Reaktion (ein Akt des Antwortens) erwartet, und umgekehrt setze ein Akt des Antwortens einen vorhergehenden Akt des Fragens voraus (vgl. Lyons 1968, 469). fragen und antworten verhalten sich auch deswegen anders als typische Konverse wie kaufen und verkaufen, weil sich ihre Thema-Argumente unterscheiden: was gefragt wird ( $\left.P^{\prime}\right)$, ist nicht identisch mit dem, was geantwortet wird (P).

Lyons schlägt noch anbieten-akzeptieren (offer-accept) und anbieten-ablehnen (offer-refuse) als mögliche Konverse vor, weist dann aber darauf hin, dass diese sich nicht wie typische Konverse, sondern vielmehr wie fragen-antworten verhalten: Zwischen den Akten, auf die mit anbieten und akzeptieren bzw. mit anbieten und ablehnen Bezug genommen wird, besteht eine Beziehung der zeitlichen Abfolge. Auf einen Akt des Anbietens wird eine positive oder negative Reaktion wie ein Akt des Akzeptierens oder des Ablehnens erwartet; umgekehrt setzt ein Akt des Akzeptierens oder des Ablehnens einen Akt des Anbietens voraus (vgl. Lyons 1968, 469). Mit Verbpaaren wie anbieten-akzeptieren und anbieten-ablehnen wird genauso wie mit fragen-antworten auf zwei aufeinanderfolgende sprachliche Handlungen Bezug genommen. Typische Konverse wie geben und nehmen oder kaufen und verkaufen werden hingegen mit Bezug auf eine einzige Handlung verwendet, die aus unterschiedlicher Perspektive dargestellt wird. Verbpaare wie fragen-antworten, anbieten-akzeptieren und anbieten-ablehnen können daher nicht als Konverse gelten.

fragen-antworten, anbieten-akzeptieren und anbieten-ablehnen können außerdem generell nicht als Gegensatzbezeichnungen betrachtet werden, weil sie aus Verben unterschiedlicher Paradigmen bestehen. Damit unterscheiden sie sich nicht nur im Hinblick auf die Unterscheidung, initial/reaktiv', sondern auch hinsichtlich der mit ihnen lexikalisierten Sprechereinstellungen wie der propositionalen Einstellung des Sprechers, der Sprecherabsicht und der Vorannahmen des Sprechers. Die Bedeutungsunterschiede zwischen den Verben dieser Paare sind damit zu groß, als dass die Verben noch als Gegensatzbezeichnungen gelten können. Ein Kontrast in Bezug auf eine oder mehrere Eigenschaften zweier Objekte wird nur dann als solcher wahrgenommen, wenn die Objekte sich in anderer Hinsicht ähnlich sind (vgl. Lyons 1977, 286). Auch wenn klar ist, dass Gegensätzlichkeit ein bestimmtes Ausmaß an Ähnlichkeit voraussetzt, ist damit noch nicht geklärt, wie unterschiedlich Gegensatzbezeichnungen sein dürfen, um noch als solche gelten zu können. Im Folgenden werden nur solche Paare von Sprechaktverben als Gegensatzbezeichnungen betrachtet, die sich nur unter dem Gesichtspunkt der Negation voneinander unterscheiden. Nach dieser engen Auffassung von Antonymie können Sprechaktverben unterschiedlicher Großparadigmen keine Gegensatzbezeichnungen sein, weil die Sprechereinstellungen, die mit ihnen lexikalisiert sind, sich niemals nur unter dem Gesichtspunkt der Negation, sondern vielmehr grundsätzlich voneinander unterscheiden. Verben wie mitteilen und verbieten kommen beispielsweise nicht als Antonyme in Betracht, obwohl die mit ihnen lexikalisierten propositionalen Einstellungen Negation involvieren: mitteilen, das zum Großparadigma der Repräsentative gehört, lexikalisiert eine kognitive Sprechereinstellung (,S kennt: $P^{\prime}$ ), während mit verbieten, das zum Großparadigma der Direktive gehört, eine voluntative Einstellung (,S will: $\left.\neg P^{\prime}\right)$ lexikalisiert ist. Da die mit diesen Verben lexikalisierten propositionalen Einstellungen sich nicht nur hinsichtlich 
der Negation, sondern auch grundsätzlich voneinander unterscheiden, liefern sie keinerlei Grundlage für die Annahme einer antonymischen Relation zwischen mitteilen und verbieten.

\subsection{Dualität}

Dualität ist eine Äquivalenzbeziehung zwischen sprachlichen Ausdrücken unter Einbeziehung von innerer, äußerer und dualer Negation, was zur Konstituierung von Dualitätsgruppen führt. Diese bestehen aus maximal vier sprachlichen Ausdrücken, sog. dualen Operatoren, die nicht notwendigerweise in einem regulären Wortzusammenhang stehen und in den meisten Fällen weder etymologisch verwandt sind noch lexikalische Antonympaare darstellen (vgl. Lenz 2002, 521). Löbner unterscheidet vier Typen von Operatoren, die den folgenden Quantoren bzw. deren Negation entsprechen: Typ 1: , Typ 2: $\forall$, Typ 3: $-\exists$ und Typ 4: $\neg \forall$ (vgl. Löbner 1990, 69-75; Lenz 2002, 521).

Die Äquivalenzbeziehungen zwischen den einzelnen Typen dualer Operatoren werden anhand eines Dualitätsdiagramms dargestellt:

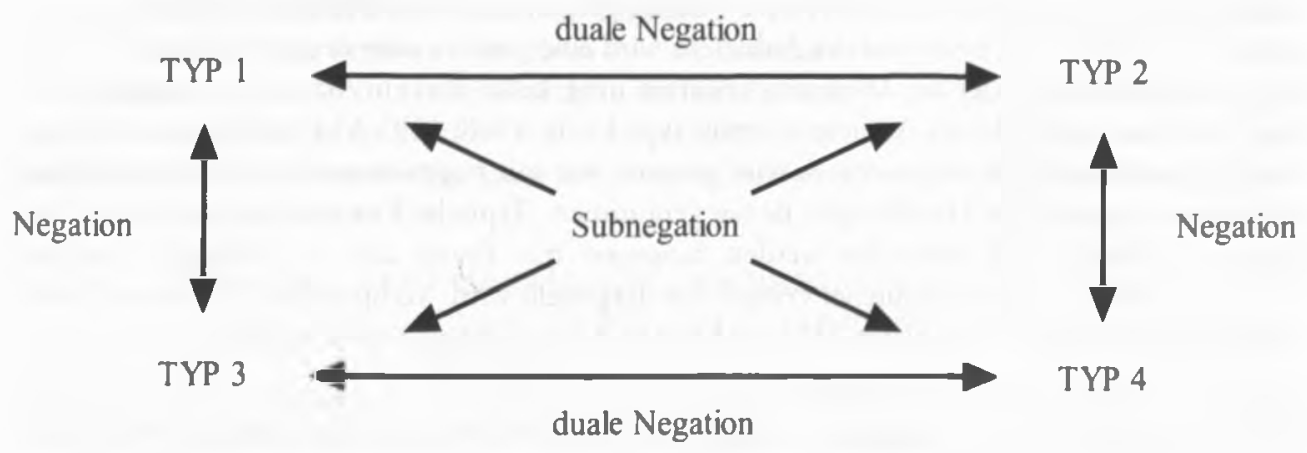

Abb. 1: Dualitätsdiagramm allgemein (nach Lenz 2002, 521)

Die im Dualitätsdiagramm dargestellten Negationstypen unterscheiden sich im Hinblick auf ihre Skopusverhältnisse: Negation steht für „äußere Negation “ und bedeutet ,Negation des Operators"; mit Subnegation (oder innerer Negation) ist die Negation des Operanden gemeint, und duale Negation bedeutet ,(äußere) Negation plus Subnegation' (vgl. Lenz 2002, 521). Lenz erläutert die drei Typen der Negation anhand der Dualitätsgruppe schon/noch nicht/noch/nicht mehr, vgl. Abb. 2: 


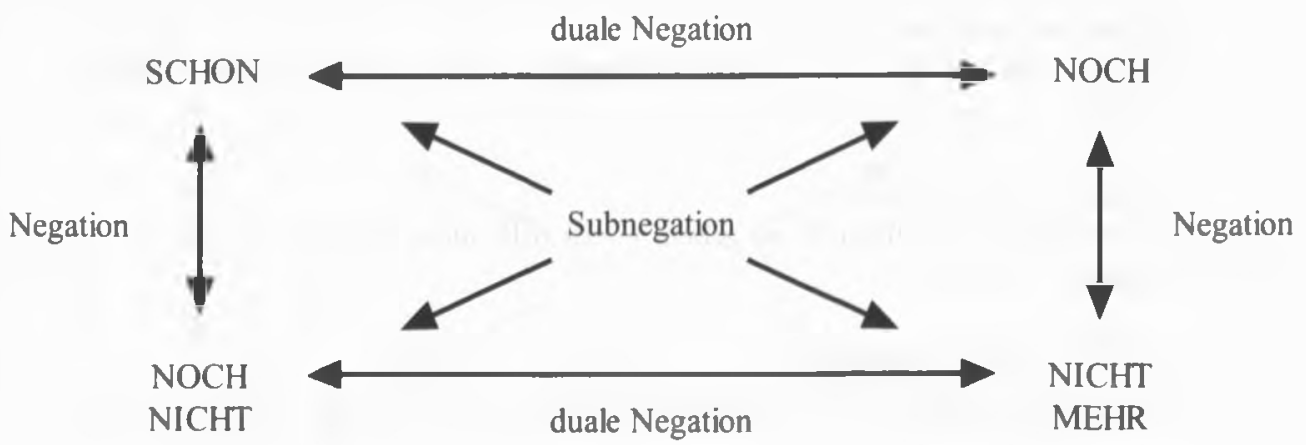

Abb. 2: Dualitätsdiagramm schon/noch nicht/noch/nicht mehr (nach Lenz 2002, 522)

Wie die Äquivalenzbeziehung zwischen den Sätzen in (9) zeigt, stehen noch und nicht mehr wie auch schon und noch nicht, in einer Beziehung der äußeren Negation:

(9) Das Licht ist NOCH an. $\leftrightarrow$ Es trifft nicht zu: Das Licht ist NICHT MEHR an.

In diesem Beispiel realisiert Es trifft nicht zu die äußere Negation.

noch und noch nicht stehen, wie auch schon und nicht mehr in einer Beziehung der Subnegation, vgl. das Äquivalenzverhältnis der Sätze in (10):

(10) Das Licht ist NOCH an. $\leftrightarrow$ Das Licht ist NOCH NICHT aus.

In diesen Sätzen wird die Subnegation durch die Antonymie von an und aus realisiert.

Die Äquivalenz der Sätze in (11) zeigt, dass noch und schon in einer Beziehung der dualen Negation (äußere Negation plus Subnegation) stehen:

(11) Das Licht ist NOCH an. $\leftrightarrow$ Es trifft nicht zu: Das Licht ist SCHON aus.

Im zweiten Satz in (11) wird die Subnegation durch die Antonymie von an und aus und die äußere Negation durch Es trifft nicht zu realisiert.

Wie die Äquivalenz der Sätze in (12) zeigt, stehen noch nicht und nicht mehr ebenfalls in einer Beziehung der dualen Negation:

(12) Das Licht ist NOCH NICHT an. $\leftrightarrow$ Es trifft nicht zu: Das Licht ist NICHT MEHR aus.

In (12) wird die Subnegation durch die Antonymie von an und aus und die äußere Negation durch Es trifft nicht $z u$ realisiert.

Zu Löbners Beispielen von Dualitätsgruppen gehören die folgenden beiden Gruppen von Sprechaktverben, die er allerdings nicht kommentiert (vgl. Löbner 1990,85 f.):

(13) Typ I: erlauben - Typ II: befehlen - Typ III: verbieten - Typ IV: $\varnothing$ (,nicht befehlen')

(14) Typ I: akzeptieren - Typ II: fordern - Typ III: ablehnen - Typ IV: verzichten auf

Wie die Äquivalenzbeziehungen der Sätze in (15) - (20) zeigen, stehen die Ausdrücke der Dualitätsgruppe in (13) (erlauben/befehlen/verbieten/ $\varnothing$ ) in unterschiedlichen Negationsbeziehungen zueinander: 
erlauben-verbieten: äußere Negation

(15) Otto hat Anna ERLAUBT, zu gehen. $\leftrightarrow$ Es trifft nicht zu: Otto hat Anna VERBOTEN, zu gehen.

befehlen- $\varnothing$ : äußere Negation

(16) Otto hat Anna BEFOHLEN, zu gehen. $\leftrightarrow$ Es trifft nicht zu: Otto hat Anna $\varnothing$ (,nicht befohlen'), zu gehen.

verbieten-befehlen: Subnegation

(17) Otto hat Anna VERBOTEN, zu gehen. $\leftrightarrow$ Otto hat Anna BEFOHLEN, zu bleiben.

erlauben- $\oslash:$ Subnegation

(18) Otto hat Anna ERLAUBT, zu gehen. $\leftrightarrow$ Otto hat Anna $\varnothing$ (,nicht befohlen'), zu bleiben.

erlauben-befeblen: duale Negation

(19) Otto hat Anna ERLAUBT, zu gehen. $\leftrightarrow$ Es trifft nicht zu: Otto hat Anna BEFOHLEN, zu bleiben.

verbieten- $\varnothing$ : duale Negation

(20) Otto hat Anna VERBOTEN, zu gehen. $\leftrightarrow$ Es trifft nicht zu: Otto hat Anna $\varnothing$ (,nicht befohlen'), zu bleiben.

Das Diagramm in Abb. 3 stellt die Negationsbeziehungen zwischen den Ausdrücken der Dualitätsgruppe erlauben/befehlen/verbieten/ $\varnothing$ dar:

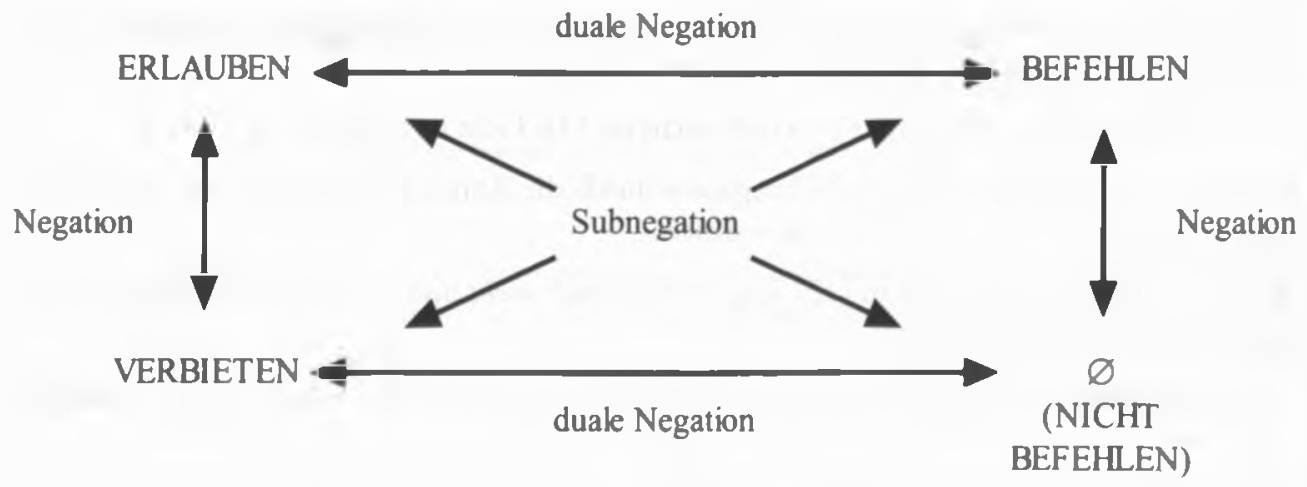

Abb. 3: Dualitätsdiagramm erlauben/befehlen/verbieten/Ø

Entsprechend können die Negationsverhältnisse zwischen den Ausdrücken der von Löbner postulierten Dualitätsgruppe in (14) (akzeptieren/fordern/ablehnen/verzichten auf) folgendermaßen dargestellt werden, wobei an der Stelle von akzeptieren, das auch mit Bezug auf einen mentalen Akt verwendet werden kann, auch Verben wie einwilligen oder zusagen stehen können, die nur eine Lesart als Sprechaktverben haben: 
AKZEPTIEREN/

EINWILLIGEN/

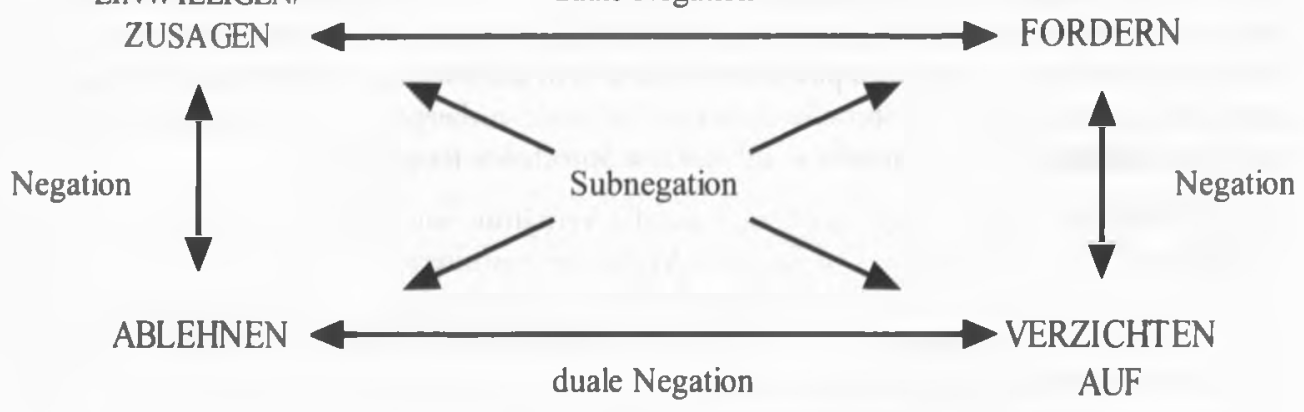

Abb. 4: Dualitätsdiagramm akzeptieren/fordernlablehnen/verzichten auf

Ob die Ausdrücke dieser Gruppe tatsächlich die für die Konstituierung einer Dualitätsgruppe notwendigen Negationsverhältnisse aufweisen, ist allerdings fraglich. Unproblematisch ist nur die Beziehung zwischen akzeptieren und ablehnen, die im Dualitätsdiagramm in Abb. 4 als eine Beziehung der äußeren Negation dargestellt ist. Die Äquivalenz der Sätze in (21) und (22) zeigt, dass akzeptieren und ablehnen sowohl in ihrer Lesart als Direktive als auch in ihrer Lesart als Kommissive in einer Beziehung der äußeren Negation stehen:

akzeptieren und ablehnen als Direktive

(21) Otto hat AKZEPTIERT, dass die Tür geschlossen wird. $\leftrightarrow$ Es trifft nicht zu: Otto hat es ABGELEHNT, dass die Tür geschlossen wird.

akzeptieren und ablehnen als Kommissive

(22) Otto hat AKZEPTIERT, länger zu arbeiten. $\leftrightarrow$ Es trifft nicht zu: Otto hat es ABGELEHNT, länger zu arbeiten.

Die für fordern und verzichten auf postulierte Beziehung der äußeren Negation setzt die semantische Äquivalenz der Särze in (23) voraus:

(23) Die Entführer FORDERTEN ein Lösegeld. - Es trifft nicht zu: Die Entführer VERZICHTETEN AUF ein Lösegeld/darauf, ein Lösegeld zu fordern.

Die Sätze in (23) sind nicht äquivalent, weil fordern ein Direktiv und verzichten auf ein Kommissiv ist. Äquivalent mit dem ersten Satz in (23) (Die Entführer forderten ein Lösegeld.) wäre ein Satz mit nicht fordern (Die Entfuhrer forderten nicht ein Lösegeld.). Ein Direktiv mit der Bedeutung ,nicht fordern' gibt es im Deutschen nicht.

Die Polysemie von Verben wie akzeptieren ist auch der Grund, warum das in Abb. 4 postulierte Verhältnis zwischen akzeptieren und verzichten problematisch ist. Da verzichten nur eine Lesart als Kommissiv, akzeptieren aber sowohl eine Lesart als Direktiv als auch eine Lesart als Kommissiv hat, könnte eine Beziehung der Subnegation grundsätzlich nur zwischen verzichten und der Lesart von akzeptieren als Kommissiv existieren. Eine Beziehung der Subnegation besteht aber auch nicht zwischen verzichten und dem Kommissiv akzeptieren, weil akzeptieren im Unterschied zu verzichten nur mit Bezug auf Sprechakte verwendet wird, die selbst eine Reaktion auf einen vorhergehenden Sprechakt sind. Ähnliches gilt für die Verben ablehnen und fordern: Da fordern nur 
eine Lesart als Direktiv, ablehnen aber sowohl eine Lesart als Direktiv als auch als Kommissiv hat, könnte es eine Beziehung der Subnegation grundsätzlich nur zwischen fordern und der Lesart von ablehnen als Direktiv geben. Eine Beziehung der Subnegation kann aber auch deswegen nicht für fordern und das Direktiv ablehnen postuliert werden, weil ablehnen nur mit Bezug auf Sprechakte verwendet werden kann, die selbst eine Reaktion auf einen vorhergehenden Sprechakt sind, während fordern nicht auf die Bezugnahme auf reaktive Sprechakte festgelegt ist.

Was für ablehnen und fordern gilt, trifft auch auf das Verhältnis von akzeptieren und fordern sowie von ablehnen und verzichten zu. Das für diese Verbpaare postulierte Verhältnis der dualen Negation kann es nur zwischen dem Direktiv akzeptieren und fordern bzw. zwischen dem Kommissiv ablehnen und verzichten geben. Für das Verbpaar ablehnen-verzichten gilt außerdem, dass mit ablehnen nur auf Sprechakte Bezug genommen werden kann, die selbst eine Reaktion auf einen vorhergehenden Sprechakt sind; verzichten ist nicht auf die Bezugnahme auf reaktive Sprechakte festgelegt. Zwischen ablehnen und verzichten besteht keine Beziehung der dualen Negation. Da die Verben der Gruppe akzeptieren/fordern/ablehnen/verzichten teilweise unterschiedlichen Großparadigmen von Sprechaktverben angehören und manche von ihnen darüber hinaus nur mit Bezug auf reaktive Äußerungen verwendet werden, bestehen zwischen ihnen nicht die Negationsbeziehungen, die für die Konstituierung einer Dualitätsgruppe notwendig sind.

Im Folgenden werden für die Großparadigmen der Repräsentative, Direktive, Kommissive und Expressive alle Fälle von Antonymie im weiteren und engeren Sinn aufgelistet. Als Antonyme im weiteren Sinn gelten Paare von Sprechaktverben, die sich außer in ihren negationsinvolvierenden Merkmalen noch in weiteren Aspekten ihrer Bedeutung unterscheiden. Unter Antonymen im engeren Sinn werden Paare von Sprechaktverben verstanden, die sich nur in denjenigen Aspekten ihrer Bedeutung unterscheiden, die Negation involvieren. Die negarionsinvolvierenden Merkmale, die für die Bestimmung einer antonymischen Relation ausschlaggebend sind, sind immer konzeptuelle Merkmale. Lexikalische Merkmale spielen für die Bestimmung einer antonymischen Relation keine Rolle; im Unterschied zu Synonymie ist Antonymie ausschließlich auf der konzeptuellen Ebene anzusiedeln.

\section{Gegensatzrelationen von Sprechaktverben}

\subsection{Gegensatzrelationen von Repräsentativen}

\subsubsection{Fälle von Antonymie im weiteren Sinn - Verben, die die propositionale Einstellung ,S hält für wahr: P’ bzw. ,S hält für wahr: - P’ lexikalisieren}

bestehen auf beharren auf pochen auf insistieren/widersprechen zurïckweisen kontern

Die Repräsentative bestehen auf, beharren auf, pochen auf und insistieren können insofern als Antonyme von widersprechen, zurückweisen und kontern betrachtet werden, als sie die propositionale Einstellung ,S hält für wahr: P' lexikalisieren, während mit Verben wie widersprechen die propositionale Einstellung ,S hält für wahr: - $\mathrm{P}^{\mathrm{P}}$ lexikalisiert ist. Verben wie bestehen auf unterscheiden sich aber auch darin von solchen wie widersprechen, dass sie mit Bezug auf Situationen verwendet werden, in denen ein Sprecher auf eine Äußerung des Hörers reagiert, die selbst eine Reaktion auf eine vorhergehende Behauptung eines Sprechers ist. Mit Verben wie widersprechen wird hingegen auf Situationen Bezug genommen, in denen ein Sprecher ablehnend auf eine 
vorausgegangene Äußerung reagiert; Verben wie bestehen auf sind re-reaktive, solche wie widersprechen reaktive Verben. Außerdem ist mit widersprechen, zurückweisen und kontern der Äußernsmodus ,deurlich' lexikalisiert, der nicht Teil der Bedeutung von Verben wie bestehen auf ist. Im Unterschied zu Verben wie bestehen auf ist mit widersprechen, zurückweisen und kontern keinerlei Vorannahme des Sprechers lexikalisiert. Da Verben wie bestehen auf und solche wie widersprechen sich nicht nur hinsichtlich der propositionalen Einstellung und der Specherabsicht (der negationsinvolvierenden Merkmale), sondern auch in weiteren Aspekten ihrer Bedeutung unterscheiden (vgl. Tab. 1), können sie nicht als Antonyme im engeren Sinn betrachtet werden. (In dieser und den nächsten Tabellen sind diejenigen Bedeutungsaspekte, in denen die Verben der aufgeführten Paradigmen sich unterscheiden, fert umrandet.)

\begin{tabular}{|c|c|c|}
\hline \multirow{2}{*}{$\begin{array}{l}\text { Ausstattung des } \\
\text { Rekurssituationstyps }\end{array}$} & \multicolumn{2}{|c|}{ Verben } \\
\hline & $\begin{array}{c}\text { bestehen auf, beharren auf, } \\
\text { pochen auf, insistieren }\end{array}$ & $\begin{array}{c}\text { widersprechen, zurückweisen, } \\
\text { kontern }\end{array}$ \\
\hline Propositionaler Gehalt (P) & Mitteilungsgehalt: P & Mitteilungsgehalt: P \\
\hline Geschehenstyp (P) & unbestimmt & unbestimmt \\
\hline Zeitbezug (P) & unbestimmt & unbestimmt \\
\hline Rollenbezug $(P)$ & unbestimmt & unbestimmt \\
\hline Einstellung von $S$ zu $P$ & S hält für wahr: $P$ & $S$ hält für wahr: $\neg P$ \\
\hline Sprecherabsicht & $\begin{array}{l}\text { S will: H erkennt: } S \text { hält für } \\
\text { wahr: P }\end{array}$ & $\begin{array}{l}S \text { will: } H \text { erkennt: } S \text { hält für } \\
\text { wahr: } \neg P\end{array}$ \\
\hline Vorannahmen von S & $\mathrm{H}$ hält für wahr: $\neg \mathrm{P}$ & - \\
\hline Äußerung & re-reaktiv & reaktiv \\
\hline Modus des Äußerns & - & deutlich \\
\hline
\end{tabular}

Tab. 1: Ausstattung der Rekurssituationstypen BESTEHEN AUF und WIDERSPRECHEN

bestehen auf beharren auf pochen auf insistieren/dementieren

bestehen auf, beharren auf, pochen auf und insistieren können insofern als Antonyme von dementieren betrachtet werden, als sie die propositionale Einstellung ,S hält für wahr: P' lexikalisieren, während mit dementieren die propositionale Einstellung,$S$ hält für wahr: $\neg P^{\prime}$ lexikalisiert ist. bestehen auf, beharren auf, pochen auf und insistieren unterscheiden sich aber auch darin von dementieren, dass sie die Vorannahme des Sprechers lexikalisieren, dass H nicht-P für wahr hält. Mit dementieren ist keinerlei Vorannahme des Sprechers lexikalisiert. dementieren lexikalisiert im Unterschied zu Verben wie bestehen auf die Spezifierung von $\mathrm{P}$ als einen öffentlich vermuteten Sachverhalt, der $\mathrm{S}$ betriff. Außerdem wird dementieren mit Bezug auf eine erste Reaktionsstufe verwendet, während mit Verben wie bestehen auf auf eine zweite Reaktionsstufe Bezug genommen wird; dementieren ist ein reaktives Prädikat; bestehen auf, beharren auf, pochen auf und insistieren sind re-reaktive Prädikate. Da Verben wie bestehen auf einerseits und dementieren andererseits sich nicht nur hinsichtlich der propositionalen Einstellung und der Sprecherabsicht (der negationsinvolvierenden Merkmale), sondern auch in weiteren Aspekten ihrer Bedeutung unterscheiden (vgl. Tab. 2), können sie nicht als Antonyme im engeren Sinn betrachtet werden. 


\begin{tabular}{|c|c|c|}
\hline \multirow{2}{*}{$\begin{array}{l}\text { Ausstattung des } \\
\text { Rekurssituationstyps }\end{array}$} & \multicolumn{2}{|c|}{ Verben } \\
\hline & $\begin{array}{c}\text { besteben auf, beharren auf, } \\
\text { pochen auf, insistieren }\end{array}$ & dementieren \\
\hline Propositionaler Gehalt (P) & Mitteilungsgehalt: P & Mitteilungsgehalt: P \\
\hline Geschehenstyp (P) & unbestimmt & unbestimmt \\
\hline Zeitbezug (P) & unbestimmt & unbestimmt \\
\hline Rollenbezug (P) & unbestimmt & unbestimmt \\
\hline Einstellung von $S$ zu $P$ & $S$ hält für wahr: $P$ & S hält für wahr: -,P \\
\hline Sprecherabsicht & $\begin{array}{l}\text { S will: } H \text { erkennt: } S \text { hält für } \\
\text { wahr: } P\end{array}$ & $\begin{array}{l}\text { S will: H erkennt: } S \text { hält für } \\
\text { wahr: }-\mathrm{P}\end{array}$ \\
\hline Vorannahmen von S & $\mathrm{H}$ hält für wahr: $\neg \mathrm{P}$ & - \\
\hline Position der Äußerung & re-reaktiv & reaktiv \\
\hline Spezifik von $\mathrm{P}$ & - & $\begin{array}{l}\text { öffentlich vermuteter Sachver- } \\
\text { halt, der } S \text { betrifft }\end{array}$ \\
\hline
\end{tabular}

Tab. 2: Ausstartung der Rekurssituationstypen BESTEHEN AUF und DEMENTIEREN

\section{bestehen aufbeharren auf pochen auf insistieren/ widerlegen entkräften}

bestehen auf, beharren auf, pochen auf und insistieren können insofern als Antonyme von widerlegen und entkräften betrachtet werden, als sie die propositionale Einstellung ,S hält für wahr: P“ lexikalisieren, während mit widerlegen und entkräften die propositionale Einstellung, $S$ hält für wahr: $\neg P^{\prime}$ lexikalisiert ist. bestehen auf, beharren auf, pochen auf und insistieren unterscheiden sich aber auch darin von widerlegen und entkräften, dass sie die Vorannahme des Sprechers lexikalisieren, dass $\mathrm{H}$ nicht-P für wahr hält. Mit widerlegen und entkräfien ist keinerlei Vorannahme des Sprechers lexikalisiert. Außerdem sind Verben wie bestehen auf re-reaktive Prädikate, während widerlegen und entkräften reaktive Prädikate sind. Letztere werden mit Bezug auf Situationen verwendet, in denen ein Sprecher $S$ auf eine Äußerung eines Hörers $\mathrm{H}$, mit der dieser P behauptet hat, reagiert und mit mehreren Äußerungen zum Ausdruck bringt, dass er nicht-P für wahr hält und dafür Gründe bzw. Argumente anführt. Dementsprechend lexikalisieren widerlegen und entkräften den Äußernsmodus, argumentariv", der nicht Teil der Bedeutung von Verben wie bestehen auf ist. Da Verben wie bestehen auf einerseits und widerlegen und entkräften andererseits sich nicht nur hinsichtlich der propositionalen Einstellung und der Sprecherabsicht (der negationsinvolvierenden Merkmale), sondern auch in weiteren Aspekten ihrer Bedeutung unterscheiden (vgl. Tab. 3), können sie nicht als Antonyme im engeren Sinn betrachtet werden. 


\begin{tabular}{|c|c|c|}
\hline \multirow{2}{*}{$\begin{array}{c}\text { Ausstattung des } \\
\text { Rekurssituationstyps }\end{array}$} & \multicolumn{2}{|c|}{ Verben } \\
\hline & $\begin{array}{c}\text { bestehen auf, beharren auf, } \\
\text { pochen auf, insistieren }\end{array}$ & widerlegen, enckräften \\
\hline Propositionaler Gehalt (P) & Mitteilungsgehalt: $P$ & Mitteilungsgehalt: $\mathbf{P}$ \\
\hline Geschehenstyp (P) & unbestimmt & unbestimmt \\
\hline Zeitbezug (P) & unbestimmt & unbestimmt \\
\hline Rollenbezug $(P)$ & unbestimmt & unbestimmt \\
\hline Einstellung von $S$ zu $P$ & $S$ hält für wahr: $P$ & $S$ hält für wahr: $\neg P$ \\
\hline Sprecherabsicht & $\begin{array}{l}S \text { will: } H \text { erkennt: } S \text { hält für } \\
\text { wahr: } P\end{array}$ & $\begin{array}{l}S \text { will: } H \text { erkennt: } S \text { hält für } \\
\text { wahr: } \neg \mathrm{P}\end{array}$ \\
\hline Vorannahmen von $S$ & H hält für wahr: $-I P$ & 一 \\
\hline Position der Äußerung & re-reaktiv & $\begin{array}{l}\text { reaktiv/Sequenz von Äußerun- } \\
\text { gen }\end{array}$ \\
\hline Äußernsmodus & - & argumentativ \\
\hline
\end{tabular}

Tab. 3: Ausstatrung der Rekurssituationstypen BESTEHEN AUF und WIDERLEGEN

\subsubsection{Fälle von Antonymie im engeren Sinn - Verben, die die propositionale Einstellung ,S hält für wahr: $\mathbf{P}^{\prime}$ bzw. ,S hält für wahr: $\neg \mathbf{P}^{\prime}$ lexikalisieren}

zustimmen beipflichten bestitigen/abstreiten bestreiten verneinen leugnen

Verben wie zustimmen bzw. solche wie abstreiten lexikalisieren die propositionale Einstellung ,S hält für wahr: P' bzw. ,S hält für wahr: $\neg P^{\prime}$ sowie die entsprechende Sprecherabsicht ,S will: $\mathrm{H}$ erkennt: S hält für wahr: P' bzw. ,S will: H erkennt: S hält für wahr: -P'. Zum zustimmen-Paradigma gehören auch die Verben beipflichten, bestätigen und bejahen; das abstreiten-Paradigma enthält auch die Verben bestreiten, verneinen und leugnen.

Da Verben wie zustimmen und abstreiten keine skalaren Ausdrücke sind, ist fraglich, ob sie als konträre Antonyme gelten können. Wenn ein Sprecher einer Behauptung eines Hörers zustimmt, streitet er sie nicht $\mathrm{ab}(\mathrm{a} \rightarrow \neg \mathrm{b}$ ), und wenn er eine Behauptung von $\mathrm{H}$ abstreitet, stimmt er ihr nicht zu $(b \rightarrow \neg a)$. Im Unterschied zu Äußerungen mit typischen Beispielen konträrer Antonyme (gut-schlecht, hoch-niedrig, lieben-hassen) können Äußerungen wie $X$ stimmt $P$ zu und $X$ streitet $P$ $a b$ nur dann beide zugleich nicht zutreffen, wenn ein Sprecher entweder gar nichts tut oder eine völlig andere Handlung vollzieht. Diese Fälle können allerdings nicht mehr der durch zustimmen und abstreiten bezeichneren Dimension des für Wahr-Haltens zugeordnet werden. Typische Beispiele konträrer Antonyme wie etwa kurz und lang können beide zugleich nicht zutreffen, ohne dass dies zu einem Dimensionswechsel führt: Wenn etwas weder lang noch kurz ist, kann es dem mittleren Bereich der Skala der möglichen Längen und damit der Dimension „Länge“ zugeordnet werden. Der Unterschied zwischen konträren Antonymen wie gut und schlecht und Antonymen wie zustimmen und widersprechen besteht darin, dass Letztere keine skalaren Ausdrücke sind; im Deutschen gibt es keine Sprechaktverben, die die Skalierung von epistemischen Einstellungen lexikalisieren. Graduierbar ist allenfalls das, was für wahr gehalten wird, d. h. P oder $\neg$ P. Sprech- 
aktverben, die die propositionale Einstellung ,S hält für wahr: eher P' lexikalisieren, gibt es im Deutschen nicht. Die propositionale Einstellung ,S hält für wahr: eher $\neg P^{‘}$ ist mit den Repräsentativen anzweifeln und bezweifeln sowie einräumen und einlenken lexikalisiert.

Verben wie zustimmen und abstreiten sind aber auch keine komplementären Antonyme. Komplementäre Antonyme wie Mann und Frau teilen einen konzeptuellen Bereich in zwei sich gegenseirig ausschließende Bereiche ein. $X$ ist ein Mann und $X$ ist eine Frau können daher nicht beide zugleich zutreffen, sowie $X$ ist kein Mann und $X$ ist keine Frau auch nicht beide zugleich zutreffen können. Während $X$ stimmt $P z u$ und $X$ streitet $P a b$ auch nicht beide zugleich zutreffen können, können $X$ stimmt $P$ nicht $z u$ und $X$ streitet $P$ nicht $a b$ aber schon zugleich zutreffen: Ein Sprecher kann etwas nicht zustimmen und es auch nicht abstreiten, indem er gar nichts tut oder eine völlig andere Handlung vollzieht.

Die Verben des zustimmen-Paradigmas und die des abstreiten-Paradigmas unterscheiden sich nur hinsichtlich der propositionalen Einstellung des Sprechers und der Sprecherabsicht, die mit ihnen lexikalisiert sind; in allen anderen Aspekten der Situationen, auf die mit ihnen Bezug genommen wird, sind sie identisch (vgl. Tab. 4). Verben wie zustimmen und solche wie abstreiten können daher als Antonyme im engeren Sinn betrachtet werden.

\begin{tabular}{|l|l|l|}
\hline \multicolumn{1}{c|}{$\begin{array}{c}\text { Ausstattung des } \\
\text { Rekurssituationstyps }\end{array}$} & \multicolumn{1}{c|}{$\begin{array}{c}\text { zustimmen, beipflichten, } \\
\text { bestätigen, bejahen }\end{array}$} & $\begin{array}{c}\text { abstreiten, bestreiten, verneinen, } \\
\text { leugnen }\end{array}$ \\
\cline { 2 - 3 } & Mitteilungsgehalt: $\mathrm{P}$ & Mitteilungsgehalt: $\mathrm{P}$ \\
\hline \hline Propositionaler Gehalt (P) & unbestimmt & unbestimmt \\
\hline Geschehenstyp (P) & unbestimmt & unbestimmt \\
\hline Zeitbezug (P) & unbestimmt & unbestimmt \\
\hline Rollenbezug (P) & S hält für wahr: P & S hält für wahr: $\neg \mathrm{P}$ \\
\hline Einstellung von S zu P & S will: $\mathrm{H}$ erkennt: S hält für & $\begin{array}{l}\text { S will: } \mathrm{H} \text { erkennt: } S \text { hält für } \\
\text { wahr: } \neg \mathrm{P}\end{array}$ \\
\hline Sprecherabsicht & wahr: P & reakriv \\
\hline Äußerung & reaktiv & \\
\hline
\end{tabular}

Tab. 4: Ausstattung der Rekurssituationstypen ZUSTIMMEN und ABSTREITEN

\subsection{Gegensatzrelationen von Direktiven}

\subsubsection{Fäle von Antonymie im weiteren Sinn}

(1) Verben, die die propositionale Einstellung ,S will: $\neg P^{\prime}$ bzw. ,S will nicht: $\neg P^{\prime}$ lexikalisieren

verbieten untersagen unterbinden verwehren einschreiten eingreifen abwürgenl erlauben gewähren gestatten

Paare von Direktiven, die aus einem Verb des verbieten-Paradigmas und einem des erlauben-Paradigmas bestehen, können insofern als Antonyme betrachtet werden, als sie gegensätzliche pro- 
positionale Einstellungen lexikalisieren: mit Verben wie verbieten ist die propositionale Einstellung ,S will: $\neg P^{\prime}$, mit Verben wie erlauben die propositionale Einstellung ,S will nicht: $-\mathrm{IP}^{\prime}$ lexikalisiert. Das verbieten-Paradigma enthält auch die Verben untersagen, unterbinden, verwehren, einschreiten, eingreifen und abwürgen. Zum erlauben-Paradigma gehören auch die Verben gewähren und gestatten.

Verben wie verbieten und solche wie erlauben unterscheiden sich aber auch hinsichtlich des Zeitbezugs von P, der Sprecherabsicht und der Vorannahmen des Sprechers. Die Verben des verbietenParadigmas lexikalisieren den Zeitbezug ,zukünftig oder gegenwärtig', während Verben wie erlauben den Zeitbezug ,zukünfrig' lexikalisieren. Mit Verben wie verbieten ist die Sprecherabsicht lexikalisiert, dass S will, dass H P nicht tur; die Verben des erlauben-Paradigmas lexikalisieren die Sprecherabsicht ,S will: H erkennt: $S$ will nicht: $H$ tut nicht: $P^{\prime}$. Mit Verben wie erlauben ist die Vorannahme des Sprechers lexikalisiert, dass P im Interesse des Hörers ist; Verben wie verbieten lexikalisieren die Vorannahme des Sprechers, dass nicht-P nicht erwartbar ist. Da die Verben des verbieten-Paradigmas und die des erlauben-Paradigmas sich nicht nur hinsichtlich der mit ihnen lexikalisierten propositionalen Einstellungen (der negationsinvolvierenden Merkmale), sondern auch im Hinblick auf weitere Aspekte ihrer Bedeutung unterscheiden, sind sie keine Antonyme im engeren Sinn (vgl. Tab. 5).

\begin{tabular}{|c|c|c|}
\hline \multirow{2}{*}{$\begin{array}{l}\text { Ausstattung des } \\
\text { Rekurssituationstyps }\end{array}$} & \multicolumn{2}{|c|}{ Verben } \\
\hline & $\begin{array}{l}\text { verbieten, untersagen, unterbin- } \\
\text { den, verwehren, einschreiten, } \\
\text { eingreifen, abwürgen }\end{array}$ & erlauben, gewähren, gestatten \\
\hline Propositionaler Gehalt (P) & Mitteilungsgehalt: $P$ & Mitteilungsgehalt: P \\
\hline Geschehenstyp (P) & Handlung & Handlung \\
\hline Zeitbezug $(P)$ & zukünftig/gegenwärtig & zukünftig \\
\hline Rollenbezug (P) & Hörer & Hörer \\
\hline Einstellung von $\mathrm{S} z \mathrm{P} P$ & S will: $\neg P$ & S will nicht: $\neg P$ \\
\hline Sprecherabsicht & S will: $H$ tut nicht: $P$ & $\begin{array}{l}S \text { will: } H \text { erkennt: } S \text { will nicht: } \\
H \text { tut nicht: } P\end{array}$ \\
\hline \multirow[t]{2}{*}{ Vorannahmen von $S$} & nicht erwartbar: $-\rightarrow P$ & - \\
\hline & - & im Interesse von $\mathrm{H}: \mathrm{P}$ \\
\hline
\end{tabular}

Tab. 5: Ausstattung der Rekurssituationstypen VERBIETEN und ERLAUBEN

\section{verbieten untersagen unterbinden verwehren einschreiten eingreifen abwürgenl einwilligen zustimmen}

Paare von Direktiven, die aus einem Verb des verbieten-Paradigmas und einem des einwilligen-Paradigmas bestehen, können insofern als Antonyme betrachtet werden, als sie die propositionale Einstellung ,S will: $\neg P^{\prime}$ bzw. ,S will nicht: $-P^{\prime}$ lexikalisieren. Zum einwilligen-Paradigma gehört noch das Verb zustimmen. Die Verben des verbieten-Paradigmas und die des einwilligen-Paradigmas unterscheiden sich aber auch hinsichtlich des Zeitbezugs von P, der Sprecherabsicht, der Vorannahmen des Sprechers und der Position der Äußerung. Während Verben wie verbieten den 
Zeitbezug ,zukünftig oder gegenwärtig' lexikalisieren, ist mit einwilligen und zustimmen der Zeitbezug ,zukünftig' lexikalisiert. Mit Verben wie verbieten ist die Sprecherabsicht ,S will: $\mathrm{H}$ tut nicht: P' lexikalisiert; Verben wie einwilligen lexikalisieren die Sprecherabsicht , $S$ will: $\mathrm{H}$ erkennt: $S$ will nicht: $\mathrm{H}$ tut nicht: P'. Im Unterschied zu Verben wie verbieten werden einwilligen und zustimmen mit Bezug auf Situationen verwendet, in denen ein Sprecher auf eine vorhergehende Äußerung des Hörers reagiert, mit der dieser zum Ausdruck bringt, dass er P tun will; einwilligen und zustimmen sind reaktive Prädikate. Darüber hinaus sind mit einwilligen und verbieten unterschiedliche Vorannahmen lexikalisiert: einwilligen lexikalisiert die Vorannahme des Sprechers, dass P im Interesse des Hörers ist; mit verbieten ist die Vorannahme des Sprechers lexikalisiert, dass $-\mathrm{P}$ nicht erwartbar ist.

Da die Verben des verbieten-Paradigmas und die des einwilligen-Paradigmas sich nicht nur hinsichtlich der mit ihnen lexikalisierten propositionalen Einstellungen (der negationsinvolvierenden Merkmale), sondern auch im Hinblick auf weitere Aspekte ihrer Bedeutung unterscheiden, sind sie keine Antonyme im engeren Sinn (vgl. Tab. 6).

\begin{tabular}{|c|c|c|}
\hline \multirow{2}{*}{$\begin{array}{l}\text { Ausstattung des } \\
\text { Rekurssituationstyps }\end{array}$} & \multicolumn{2}{|c|}{ Verben } \\
\hline & $\begin{array}{l}\text { verbieten, untersagen, unterbin- } \\
\text { den, verwehren, einschreiten, } \\
\text { eingreifen, abwürgen }\end{array}$ & einwilligen, zustimmen \\
\hline Propositionaler Gehalt (P) & Mitteilungsgehalt: $P$ & Mitteilungsgehalt: $\mathrm{P}$ \\
\hline Geschehenstyp (P) & Handlung & Handlung \\
\hline Zeitbezug (P) & zukünftig/gegenwärtig & zukünftig \\
\hline Rollenbezug (P) & Hörer & Hörer \\
\hline Einstellung von $\mathrm{S}$ zu $\mathrm{P}$ & $S$ will: $\neg P$ & S will nicht: $\neg P$ \\
\hline Sprecherabsicht & $S$ will: $\mathrm{H}$ tut nicht: $P$ & $\begin{array}{l}\text { S will: } H \text { erkennt: } S \text { will nicht: } \\
\mathrm{H} \text { tut nicht: } \mathrm{P}\end{array}$ \\
\hline \multirow[t]{2}{*}{ Vorannahmen von $S$} & nicht erwartbar: $\neg P$ & - \\
\hline & - & im Interesse von $\mathrm{H}$ : $\mathrm{P}$ \\
\hline Äußerung & - & reaktiv \\
\hline
\end{tabular}

Tab. 6: Ausstattung der Rekurssituationstypen VERBIETEN und EINWILLIGEN
verbieten untersagen unterbinden verwehren einschreiten eingreifen abwürgenl genehmigen bewilligen

Paare von Direktiven, die aus einem Verb des verbieten-Paradigmas und einem des genehmigenParadigmas bestehen, können insofern als Antonyme betrachtet werden, als sie die propositionale Einstellung ,S will: ᄀP' bzw. ,S will nicht: $\rightarrow P^{\prime}$ lexikalisieren. Zum genehmigen-Paradigma gehört auch das Verb bewilligen. Die Verben des verbieten-Paradigmas und die des genehmigen-Paradigmas unterscheiden sich aber auch hinsichtlich des Zeitbezugs von P, der Sprecherabsicht, der Position der Äußerung, der Rollenspezifik, der Vorannahmen des Sprechers und des Äußernsmodus. Während Verben wie verbieten den Zeitbezug ,gegenwärtig oder zukünftig' lexikalisieren, 
ist mit genehmigen und bewilligen der Zeitbezug ,zukünftig' lexikalisiert. Mit Verben wie verbieten ist die Sprecherabsicht ,S will: $\mathrm{H}$ tut nicht: P' lexikalisiert; genehmigen und bewilligen lexikalisieren die Sprecherabsicht ,S will: H erkennt: $S$ will nicht: H tut nicht: P'. Im Unterschied zu den Verben des verbieten-Paradigmas sind genehmigen und bewilligen reaktive Prädikate: sie werden mit Bezug auf Situationen verwendet, in denen ein Sprecher als Reaktion auf die Kundgabe des Hörers, P tun zu wollen, zum Ausdruck bringt, dass er nicht will, dass der Hörer P nicht tut. S ist weisungsbefugt oder eine Autoritätsperson (Rollenspezifik). Verben wie verbieten lexikalisieren die Vorannahme des Sprechers, dass $\neg \mathrm{P}$ nicht erwartbar ist; mit genehmigen und bewilligen ist die Vorannahme des Sprechers lexikalisiert, dass P im Interesse von H ist. Im Unterschied zu den Verben des verbieten-Paradigmas lexikalisieren genehmigen und bewilligen den Äußernsmodus ,offiziell'; sie werden mit Bezug auf ein offizielles Verfahren oder einen offiziellen Rahmen verwendet. Da die Verben des verbieten-Paradigmas und die des genehmigen-Paradigmas sich nicht nur hinsichtlich der mit ihnen lexikalisierten propositionalen Einstellungen (der negationsinvolvierenden Merkmale), sondern auch in zahlreichen anderen Aspekten ihrer Bedeutung unterscheiden, können sie nicht als Antonyme im engeren Sinn betrachtet werden (vgl. Tab. 7). (In der Kollokation einen Antrag bewilligen kann bewilligen durch das antonymische Verb abweisen ersetzt werden, um die Kollokation einen Antrag abweisen zu bilden.)

\begin{tabular}{|c|c|c|}
\hline \multirow{2}{*}{$\begin{array}{l}\text { Ausstattung des } \\
\text { Rekurssituationstyps }\end{array}$} & \multicolumn{2}{|c|}{ Verben } \\
\hline & $\begin{array}{l}\text { verbieten, untersagen, unterbin- } \\
\text { den, verwehren, einschreiten, } \\
\text { eingreifen, abwürgen }\end{array}$ & genehmigen, bewilligen \\
\hline Propositionaler Gehalt (P) & Mitteilungsgehalt: P & Mitteilungsgehalt: P \\
\hline Geschehenstyp (P) & Handlung & Handlung \\
\hline Zeitbezug (P) & zukünftig/gegenwärtig & zukünftig \\
\hline Rollenbezug (P) & Hörer & Hörer \\
\hline Einstellung von $\mathrm{S}$ zu $\mathrm{P}$ & S will: $\neg P$ & S will nicht: $\neg P$ \\
\hline Sprecherabsicht & $S$ will: $H$ tut nicht: $P$ & $\begin{array}{l}\text { S will: } H \text { erkennt: } S \text { will nicht: } \\
H \text { tut nicht: } P\end{array}$ \\
\hline \multirow[t]{2}{*}{ Vorannahmen von $S$} & nicht erwartbar: $\neg P$ & - \\
\hline & 一 & im Interesse von $\mathrm{H}: \mathrm{P}$ \\
\hline Position der Äußerung & 一 & reaktiv \\
\hline Rollenspezifik & & $S$ ist weisungsbefugt \\
\hline Äußernsmodus/Verfahren & & offiziell \\
\hline
\end{tabular}

Tab. 7: Ausstattung der Rekurssituationstypen VERBIETEN und GENEHMIGEN

(2) Verben, die die propositionale Einstellung ,S will: P’ bzw. ,S will: - $P$ ' lexikalisieren

bestellen abonnieren reservieren vorbestellen ordernlabbestellen absagen annullieren kündigen zurücktreten abblasen zurïcknehmen 
Direktive wie bestellen bzw, solche wie abbestellen können insofern als Antonyme betrachtet werden, als sie gegensätzliche propositionale Einstellungen und gegensätzliche Sprecherabsichten lexikalisieren: Verben wie bestellen lexikalisieren die propositionale Einstellung ,S will: P' und die Sprecherabsicht ,S will: $\mathrm{H}$ tur: P'; mit Verben wie abbestellen sind die propositionale Einstellung ,S will: $\neg P^{\prime}$ und die Sprecherabsicht ,S will: $\mathrm{H}$ tut nicht: $\mathrm{P}^{\prime}$ lexikalisiert. Zum bestellen-Paradigma gehören auch die Verben abonnieren, reservieren, vorbestellen und ordern; das abbestellen-Paradigma enthält auch die Verben absagen, annullieren, kündigen, zurücktreten, abblasen und zurücknehmen.

Die Verben des bestellen-Paradigmas und die des abbestellen-Paradigmas unterscheiden sich aber auch hinsichtlich der Spezifierung von P: Verben wie bestellen lexikalisieren die Spezifizierung von P als Gegenstand einer Dienstleistung, die des abbestellen-Paradigmas die Spezifizierung von $P$ als Gegenstand einer vorausgegangenen Vereinbarung. Da die Verben des bestellen-Paradigmas und die des abbestellen-Paradigmas sich nicht nur hinsichtlich der propositionalen Einstellung und der Sprecherabsicht (der negationsinvolvierenden Merkmale), sondern auch in einem weiteren Aspekt ihrer Bedeutung unterscheiden, können sie nicht als Antonyme im engeren Sinn betrachtet werden (vgl. Tab. 8).

\begin{tabular}{|l|l|l|}
\hline \multicolumn{1}{|c|}{$\begin{array}{c}\text { Ausstattung des } \\
\text { Rekurssituationstyps }\end{array}$} & $\begin{array}{c}\text { Vestellen, abonnieren, reservie- } \\
\text { ren, vorbestellen, ordern }\end{array}$ & $\begin{array}{c}\text { abbestellen, absagen, annullie- } \\
\text { ren, kündigen, zurücktreten, } \\
\text { abblasen, zurïcknehmen }\end{array}$ \\
\cline { 2 - 3 } & Mitteilungsgehalt: P & Mitteilungsgehalt: P \\
\hline \hline Propositionaler Gehalt (P) & Handlung & Handlung \\
\hline Geschehenstyp (P) & zukünftig & zukünftig \\
\hline Zeitbezug (P) & Hörer & Hörer \\
\hline Rollenbezug (P) & S will: P & S will: $\neg P$ \\
\hline Einstellung von S zu P & S will: H tut: P & S will: H tut nicht: P \\
\hline Sprecherabsicht & P ist im Aufgaben-/Kom- & $\begin{array}{l}\text { P ist im Aufgaben-/Kom- } \\
\text { petenzbereich von H }\end{array}$ \\
\hline Vorannahmen von S & Getenzbereich von H & $\begin{array}{l}\text { Gegenstand einer voraus- } \\
\text { gegangenen Vereinbarung }\end{array}$ \\
\hline Spezifizierung von P & tung &
\end{tabular}

Tab. 8: Ausstattung der Rekurssituationstypen BESTELLEN und ABBESTELLEN

\subsubsection{Fälle von Antonymie im engeren Sinn}

Keine 


\subsection{Gegensatzrelationen von Kommissiven}

\subsubsection{Fälle von Antonymie im weiteren Sinn}

(1) Verben, die die propositionale Einstellung ,S will: $\mathrm{P}$ tun' bzw. ,S will: nicht $\mathrm{P}$ tun` lexikalisieren

einwilligen zusagen/ablehnen zurückweisen

Paare von Kommissiven, die aus einem Verb des einwilligen-Paradigmas und einem des ablehnenParadigmas bestehen, können insofern als Antonyme betrachtet werden, als sie gegensätzliche propositionale Einstellungen und gegensätzliche Sprecherabsichten lexikalisieren: mit Verben wie einwilligen ist die propositionale Einstellung ,S will: P tun' und die Sprecherabsicht ,S will: $\mathrm{H}$ erkennt: $S$ will: $P$ tun' lexikalisiert, während Verben wie ablehnen die propositionale Einstellung ,S will nicht: P tun' und die Sprecherabsicht ,S will: $\mathrm{H}$ erkennt: S will nicht: P tun' lexikalisieren. In beiden Fällen ist P eine zukünftige Handlung des Sprechers. Zum einwilligen-Paradigma gehört auch das Verb zusagen; das ablehnen-Paradigma enthält auch das Verb zurückweisen. Verben wie einwilligen und solche wie ablehnen sind reaktive Kommissive: sie werden mit Bezug auf Situationen verwendet, in denen ein Sprecher als Reaktion auf eine Forderung oder eine vorangegangene Äußerung zum Ausdruck bringt, dass er P (nicht) tun will.

einwilligen und zusagen unterscheiden sich nicht nur hinsichtlich der propositionalen Einstellung und der Sprecherabsicht von ablehnen und zurückweisen, sondern auch darin, dass sie die Vorannahme des Sprechers lexikalisieren, dass $\mathrm{P}$ im Interesse von $\mathrm{H}$ ist. Mit ablehnen und zurückweisen ist keinerlei Vorannahme des Sprechers lexikalisiert. Da die Verben des einwilligen-Paradigmas und die des zurückweisen-Paradigmas sich in mehr als nur den negationsinvolvierenden Merkmalen unterscheiden, können sie nicht als Antonyme im engeren Sinn betrachtet werden (vgl. Tab. 9).

\begin{tabular}{|l|l|l|}
\hline \multirow{2}{*}{$\begin{array}{c}\text { Ausstattung des } \\
\text { Rekurssituationstyps }\end{array}$} & \multicolumn{1}{c|}{ einwilligen, zusagen } & \multicolumn{1}{c|}{ ablehnen, zurückweisen } \\
\cline { 2 - 3 } & Mitteilungsgehalt: P & Mitteilungsgehalt: P \\
\hline \hline Propositionaler Gehalt (P) & Handlung & Handlung \\
\hline Geschehenstyp (P) & zukünftig & zukünftig \\
\hline Zeitbezug (P) & Sprecher & Sprecher \\
\hline Rollenbezug (P) & S will: P tun & S will nicht: P tun \\
\hline Einstellung von S zu P & S will: H erkennt: S will: P tun & $\begin{array}{l}\text { S will: H erkennt: S will nicht: } \\
\text { P tun }\end{array}$ \\
\hline Sprecherabsicht & & - \\
\hline Vorannahmen von S & im Interesse von H: P & reaktiv \\
\hline Äußerung & reaktiv & \\
\hline
\end{tabular}

Tab. 9: Ausstattung der Rekurssituationstypen EINWILLIGEN und ABLEHNEN 
(2) Verben, die die Vorannahme, im Interesse von $\mathrm{H}: \mathrm{P}^{\prime}$ bzw, nicht im Interesse von $\mathrm{H}: \mathrm{P}^{\prime}$ lexikalisieren

versprechen versichern zusichern/drohen androhen

Paare von Kommissiven, die aus einem Verb des versprechen-Paradigmas und einem des drohenParadigmas bestehen, können insofern als Antonyme betrachtet werden, als sie gegensätzliche Vorannahmen des Sprechers lexikalisieren: mit Verben wie versprechen ist die Vorannahme des Sprechers lexikalisiert, dass $\mathrm{P}$ im Interesse von $\mathrm{H}$ ist; die Verben des drohen-Paradigmas lexikalisieren die Vorannahme des Sprechers, dass P nicht im Interesse von H ist. Zum versprechen-Paradigma gehören auch die Verben versichern und zusichern; das drohen-Paradigma enthält auch das Verb androhen.

Während die Bedeutung von versprechen beschrieben werden kann als, ankündigen, etwas zu tun, was im Interesse von $\mathrm{H}$ ist', deckt die entsprechende Paraphrase, ankündigen, etwas zu tun, was nicht im Interesse von $\mathrm{H}$ ist' die Bedeutung von drahen nur unvollständig ab. Verben wie drohen werden mit Bezug auf Situationen verwendet, in denen ein Sprecher zum Ausdruck bringt, dass er etwas tun will, das nicht im Interesse von $\mathrm{H}$ ist, wenn $\mathrm{H}$ nicht bestimmte Bedingungen $\mathrm{C}$ erfüllt. Mit Verben wie drohen sind die propositionale Einstellung , $\mathrm{S}$ will: $\mathrm{P}$ tun, wenn nicht $\mathrm{C}^{\prime}$ und die Sprecherabsicht ,S will: H erkennt: $S$ will: P tun, wenn nicht C' lexikalisiert; Verben wie versprechen lexikalisieren die propositionale Einstellung ,S will: P tun' und die Sprecherabsicht ,S will: H erkennt: S will: P tun'. Verben wie drohen unterscheiden sich auch darin von solchen wie versprechen, dass mit ihnen die Vorannahme lexikalisiert ist, dass $\mathrm{C}$ nicht erwartbar ist (vgl. Tab. 10). Da die Bedeutung von Verben wie drohen komplexer ist als die von Verben wie versprechen, können die Verben des versprechen-Paradigmas und die des drohen-Paradigmas trotz der gegensätzlichen Vorannahmen, die mit ihnen lexikalisiert sind, nicht als Antonyme im engeren Sinn betrachtet werden.

\begin{tabular}{|l|l|l|}
\hline \multirow{2}{*}{$\begin{array}{c}\text { Ausstattung des } \\
\text { Rekurssituationstyps }\end{array}$} & \multicolumn{1}{c|}{$\begin{array}{c}\text { Versprechen, versichern, } \\
\text { zusichern }\end{array}$} & \multicolumn{1}{c|}{ drohen, androhen } \\
\cline { 2 - 3 } & \multicolumn{1}{c|}{$\begin{array}{c}\text { Mitteilungsgehalt: } \mathrm{P} \\
\text { Propositionaler Gehalt (P) }\end{array}$} & Mitteilungsgehalt: P \\
\hline Geschehenstyp (P) & Handlung & Handlung \\
\hline Zeitbezug (P) & zukünftig & zukünfrig \\
\hline Rollenbezug (P) & Sprecher & Sprecher \\
\hline Einstellung von S zu P & S will: P tun & S will: P tun, wenn nicht C \\
\hline Sprecherabsicht & S will: H erkennt: S will: P tun & $\begin{array}{l}\text { S will: H erkennt: S will: } P \\
\text { tun, wenn nicht C }\end{array}$ \\
\hline Vorannahmen von S & im Interesse von H: P & nicht im Interesse von H: P \\
\hline & - & nicht erwartbar: C \\
\hline
\end{tabular}

Tab. 10: Ausstattung der Rekurssituationstypen VERSPRECHEN und DROHEN 


\subsubsection{Fälle von Antonymie im engeren Sinn}

Keine

\subsection{Gegensatzrelationen von Expressiven}

\subsubsection{Fälle von Antonymie im weiteren Sinn}

(1) Verben, die die propositionale Einstellung, S findet: P gut' bzw. ,S findet: P schlecht" lexikalisieren

gutheißen begrüßßen bejahen befürworten anerkennen/tadeln rügen rüffeln kritisieren beanstanden bemängeln monieren missbilligen verurteilen anprangern schelten

Die Verben des gutheißen-Paradigmas und die des tadeln-Paradigmas können insofern als Antonyme betrachtet werden, als sie gegensätzliche propositionale Einstellungen und gegensätzliche Sprecherabsichten lexikalisieren. Mit Verben wie gutheißen ist die propositionale Einstellung ,S findet: P gut' und die Sprecherabsicht ,S will: H erkennt: S findet: P gut' lexikalisiert; Verben wie tadeln lexikalisieren die propositionale Einstellung ,S findet: P schlecht' und die Sprecherabsicht „S will: H erkennt: S finder: P schlecht'. Zum gutheißen-Paradigma gehören auch die Verben begrüßen, bejahen, befürworten und anerkennen. Das tadeln-Paradigma enthält auch die Verben rügen, rüffeln, kritisieren, beanstanden, bemängeln, monieren, missbilligen, verurteilen, anprangern und schelten.

Die Verben des gutheißen-Paradigmas und die des tadeln-Paradigmas unterscheiden sich aber auch hinsichtlich des Geschehenstyps, des Zeirbezugs und des Rollenbezugs von P: Im Unterschied zu Verben wie tadeln sind die Verben des gutheißen-Paradigmas hinsichtlich dieser Aspekte nicht spezifiziert. Während Verben wie tadeln mit Bezug auf Situationen verwendet werden, in denen ein Sprecher eine (negative) Bewertung einer vergangenen Handlung von $\mathrm{H}$ oder eines Dritten zum Ausdruck bringt, wird mit Verben wie gutheißen auf Situationen Bezug genommen, in denen ein Sprecher eine (positive) Bewertung eines vergangenen, gegenwärtigen oder zukünftigen P (einer Handlung, eines Zustands oder eines Ereignisses) äußert. Da die Verben des gutheißsen-Paradigmas und die des tadeln-Paradigmas sich nicht nur hinsichtlich der propositonalen Einstellung und der Sprecherabsicht (der negationsinvolvierenden Merkmale), sondern auch in weiteren Aspekten ihrer Bedeutung unterscheiden, sind sie keine Antonyme im engeren Sinn (vgl. Tab. 11). 


\begin{tabular}{|c|c|c|}
\hline \multirow{2}{*}{$\begin{array}{l}\text { Ausstattung des } \\
\text { Rekurssituationstyps }\end{array}$} & \multicolumn{2}{|c|}{ Verben } \\
\hline & $\begin{array}{c}\text { gutheißen, begrüßen, bejahen, } \\
\text { befürworten, anerkennen }\end{array}$ & $\begin{array}{l}\text { tadeln, rügen, rüffeln, kritisie- } \\
\text { ren, beanstanden, bemängeln, } \\
\text { monieren, missbilligen, ver- } \\
\text { urteilen, anprangern, schelten }\end{array}$ \\
\hline Propositionaler Gehalt (P) & Mitteilungsgehalt: $\mathrm{P}$ & Mitteilungsgehalt: $P$ \\
\hline Geschehenstyp (P) & unbestimmt & Handlung \\
\hline Zeitbezug (P) & unbestimmt & vergangen \\
\hline Rollenbezug (P) & unbestimmt & Hörer oder Dritte \\
\hline Einstellung von $S$ zu P & $S$ findet: $P$ gut & S findet: P schlecht \\
\hline Sprecherabsicht & $\begin{array}{l}\text { S will: } H \text { erkennt: } S \text { findet: } P \\
\text { gut }\end{array}$ & $\begin{array}{l}\text { S will: H erkennt: } S \text { findet: } P \\
\text { schlecht }\end{array}$ \\
\hline Vorannahmen von S & $P$ ist der Fall & $P$ ist der Fall \\
\hline
\end{tabular}

Tab. 11: Ausstattung der Rekurssituationstypen GUTHEISSEN und TADELN

preisen lobpreisen rühmen schwärmen/ tadeln rügen rüffeln kritisieren beanstanden bemängeln monieren missbilligen verurteilen anprangern schelten

Wie die Verben des gutheißen-Paradigmas lexikalisieren auch die des preisen-Paradigmas die propositionale Einstellung ,S findet: P gut' und die Sprecherabsicht ,S will: H erkennt: S findet: P gut'. Zum preisen-Paradigma gehören auch die Verben lobpreisen, rühmen und schwärmen. Die Verben des preisen-Paradigmas können dennoch nicht als Antonyme von Verben wie tadeln betrachtet werden, weil sie sich auch hinsichtlich des Geschehenstyps und des Rollenbezugs von P sowie im Hinblick auf den mit ihnen lexikalisierten Äußernsmodus von diesen unterscheiden. Während Verben wie tadeln mit Bezug auf Situationen verwendet werden, in denen ein Sprecher eine negative Bewertung einer vergangenen Handlung von $\mathrm{H}$ oder eines Dritten zum Ausdruck bringt, werden Verben wie preisen zur Bezugnahme auf Situationen verwendet, in denen ein Sprecher überschwänglich zum Ausdruck bringt, dass er ein vergangenes P (eine Handlung, einen Zustand oder ein Ereignis) positiv bewertet. Da die Verben des preisen-Paradigmas und die des tadeln-Paradigmas sich nicht nur hinsichtlich der propositionalen Einstellung und der Sprecherabsicht (der negationsinvolvierenden Merkmale), sondern auch im Hinblick auf weitere Aspekte ihrer Bedeutung unterscheiden, können sie nicht als Antonyme im engeren Sinn betrachtet werden (vgl. Tab. 12). 


\begin{tabular}{|c|c|c|}
\hline \multirow{2}{*}{$\begin{array}{c}\text { Ausstattung des } \\
\text { Rekurssituationstyps }\end{array}$} & \multicolumn{2}{|c|}{ Verben } \\
\hline & $\begin{array}{l}\text { preisen, lobpreisen, rühmen, } \\
\text { schwärmen }\end{array}$ & $\begin{array}{l}\text { tadeln, rügen, rüffeln, kritisie- } \\
\text { ren, beanstanden, bemängeln, } \\
\text { monieren, missbilligen, ver- } \\
\text { urteilen, anprangern, schelten }\end{array}$ \\
\hline Propositionaler Gehalt (P) & Mitteilungsgehalt: $P$ & Mitteilungsgehalt: $P$ \\
\hline Geschehenstyp (P) & unbestimmt & Handlung \\
\hline Zeitbezug $(P)$ & vergangen & vergangen \\
\hline Rollenbezug (P) & unbestimmt & Hörer oder Dritte \\
\hline Einstellung von $S$ zu $P$ & $S$ findet: $P$ gut & $S$ findet: P schlecht \\
\hline Sprecherabsicht & $\begin{array}{l}S \text { will: } H \text { erkennt: } S \text { findet: } P \\
\text { gut }\end{array}$ & $\begin{array}{l}\text { S will: H erkennt: } S \text { findet: P } \\
\text { schlecht }\end{array}$ \\
\hline Vorannahmen von $S$ & $P$ ist der Fall & $P$ ist der Fall \\
\hline Äußernsmodus & überschwänglich & - \\
\hline
\end{tabular}

Tab. 12: Ausstartung der Rekurssituationstypen PREISEN und TADELN

loben würdigen honorieren huldigen ehren/meckern mosern motzen nörgeln murren mäkeln granteln beklagen sich beklagen sich beschweren

Die Verben des loben-Paradigmas und die des meckern-Paradigmas kommen insofern als Antonyme in Frage, als mit ihnen gegensätzliche propositionale Einstellungen und gegensätzliche Sprecherabsichten lexikalisiert sind. Mit Verben wie loben ist die propositionale Einstellung ,S findet: P gut' und die Sprecherabsicht, $S$ will: $\mathrm{H}$ erkennt: $S$ findet: P gut' lexikalisiert; Verben wie meckern lexikalisieren die propositionale Einstellung ,S findet: Pschlecht' und die Sprecherabsicht ,S will: H erkennt: S findet: P schlecht'. Zum loben-Paradigma gehören auch die Verben würdigen, honorieren, huldigen und ehren. Das meckern-Paradigma enthält auch die Verben mosern, motzen, nörgeln, murren, mäkeln, granteln, beklagen, sich beklagen und sich beschweren.

Die Verben des loben-Paradigmas und die des meckern-Paradigmas unterscheiden sich aber auch hinsichtlich des Geschehenstyps und des Rollenbezugs von P: Verben wie meckern sind hinsichtlich des Geschehenstyps (und damit auch des Rollenbezugs) von P nicht spezifiziert; Verben wie loben lexikalisieren den Geschehenstyp ,Handlung' und den Rollenbezug ,Hörer oder Dritté. Da die Verben des loben-Paradigmas und die des meckern-Paradigmas sich nicht nur hinsichtlich der propositionalen Einstellung und der Sprecherabsicht (der negationsinvolvierenden Merkmale), sondern auch im Hinblick auf andere Aspekte ihrer Bedeutung unterscheiden, sind sie keine Antonyme im engeren Sinn (vgl. Tab. 13). 


\begin{tabular}{|l|l|l|}
\hline \multirow{2}{*}{$\begin{array}{c}\text { Ausstattung des } \\
\text { Rekurssituationstyps }\end{array}$} & \multicolumn{1}{c|}{$\begin{array}{c}\text { Verben } \\
\text { buldigen, ehren }\end{array}$} & $\begin{array}{c}\text { meckern, mosern, motzen, nör- } \\
\text { geln, murren, mäkeln, granteln, } \\
\text { beklagen, sich beklagen, sich } \\
\text { beschweren }\end{array}$ \\
\hline \hline Propositionaler Gehalt (P) & Mitteilungsgehalt: P & Mitteilungsgehalt: P \\
\hline Geschehenstyp (P) & Handlung & unbestimmt \\
\hline Zeitbezug (P) & vergangen & vergangen \\
\hline Rollenbezug (P) & Hörer oder Dritte & unbestimmt \\
\hline Einstellung von S zu P & S findet: P gut & S findet: P schlecht \\
\hline Sprecherabsicht & S will: H erkennt: S findet: P \\
gut & $\begin{array}{l}\text { S will: } \text { H erkennt: S findet: } P \\
\text { schlecht }\end{array}$ \\
\hline Vorannahmen von S & P ist der Fall & P ist der Fall \\
\hline
\end{tabular}

Tab. 13: Ausstattung der Rekurssituationstypen LOBEN und MECKERN

\section{loben würdigen honorieren huldigen ehren/vorwerfen vorhalten zurechtweisen}

Schließlich kommen noch die Verben des vorwerfen-Paradigmas als Antonyme von Verben wie loben in Betracht. Zum vorwerfen-Paradigma gehören auch die Verben vorhalten und zurechtweisen. Obwohl Verben wie vorwerfen die propositionale Einstellung ,S findet: $P$ schlecht ${ }^{6}$ und die Sprecherabsicht ,S will: H erkennt: S findet: P schlecht' lexikalisieren, können sie dennoch nicht als Antonyme im engeren Sinn von Verben wie loben gelten, weil sie sich außerdem hinsichtlich des Rollenbezugs von P und des mit ihnen lexikalisierten Äußernsmodus von diesen unterscheiden. Mit Verben wie vorwerfen wird auf Situationen Bezug genommen, in denen ein Sprecher nachdrücklich eine negative Bewertung einer vergangenen Handlung von $\mathrm{H}$ äußert. Verben wie loben werden mit Bezug auf eine vergangene Handlung eines Hörers oder eines Dritten verwendet. Der Äußernsmodus ,nachdrücklich“ ist nicht Teil der Bedeutung von Verben wie loben. Da die Verben des loben-Paradigmas und die des vorwerfen-Paradigmas sich auch in anderen als den negationsinvolvierenden Merkmalen unterscheiden, können sie nicht als Antonyme im engeren Sinn betrachtet werden (vgl. Tab. 14). 


\begin{tabular}{|c|c|c|}
\hline \multirow{2}{*}{$\begin{array}{c}\text { Ausstattung des } \\
\text { Rekurssituationstyps }\end{array}$} & \multicolumn{2}{|c|}{ Verben } \\
\hline & $\begin{array}{c}\text { Loben, würdigen, honorieren, } \\
\text { huldigen, ehren }\end{array}$ & $\begin{array}{l}\text { vorwerfen, vorhalten, } \\
\text { zurechtweisen }\end{array}$ \\
\hline Propositionaler Gehalt (P) & Mitteilungsgehalt: $\mathrm{P}$ & Mitteilungsgehalt: $P$ \\
\hline Geschehenstyp (P) & Handlung & Handlung \\
\hline Zeitbezug (P) & vergangen & vergangen \\
\hline Rollenbezug (P) & Hörer oder Dritte & Hörer \\
\hline Einstellung von $S$ zu $P$ & $S$ findet: $P$ gut & $S$ findet: P schlecht \\
\hline Sprecherabsicht & $\begin{array}{l}\text { S will: H erkennt: } S \text { findet: } P \\
\text { gut }\end{array}$ & $\begin{array}{l}\text { S will: } H \text { erkennt: } S \text { findet: } P \\
\text { schlecht }\end{array}$ \\
\hline Vorannahmen von $S$ & $P$ ist der Fall & $P$ ist der Fall \\
\hline Äußernsmodus & - & nachdrücklich \\
\hline
\end{tabular}

Tab. 14: Ausstattung der Rekurssituationstypen LOBEN und VORWERFEN

(2) Verben, die die propositionale Einstellung ,S empfindet: Freude wegen P’ bzw. „S empfindet: Ärger/Leid wegen P' lexikalisieren

Wenn das Empfinden von Freude als ein Gemütszustand aufgefasst wird, der als positiv bewertet wird, und das Empfinden von Ärger oder Leid als ein Gemütszustand, der als negativ gilt, können Freude und Leid bzw. Ärger als Gegensätze betrachtet werden. Als Antonyme kämen dann auch Verbpaare in Betracht, mit denen auf die Kundgabe dieser Emotionen Bezug genommen wird. Beispiele solcher Verbpaare sind jubeln-klagen und jubeln-schimpfen.

jubeln jubilieren frohlocken jauchzen zujubeln/klagen wehklagen sich beklagen
sich beschweren bedauern jammern lamentieren vorklagen vorjammern vorbeulen

Wenn Emotionen wie Freude und Leid als Gegensärze aufgefasst werden, können die Verben des jubeln-Paradigmas und die des klagen-Paradigmas als Antonyme betrachtet werden: Mit Verben wie jubeln ist die propositionale Einstellung ,S empfindet: Freude wegen P' und die Sprecherabsicht , $S$ will: $H$ erkennt: $S$ empfindet: Freude wegen $P^{\prime}$ lexikalisiert; Verben wie klagen lexikalisieren die propositionale Einstellung ,S empfindet: Leid wegen P' und die Sprecherabsicht , $S$ will: $\mathrm{H}$ erkennt: $S$ empfindet: Leid wegen $P^{\prime}$. Zum jubeln-Paradigma gehören auch die Verben jubilieren, frohlocken, jauchzen und zujubeln. Das klagen-Paradigma enthält auch die Verben wehklagen, sich beklagen, sich beschweren, bedauern, jammern, lamentieren, vorklagen, vorjammern und vorheulen.

Die Verben des jubeln-Paradigmas unterscheiden sich aber auch darin von denen des klagen-Paradigmas, dass sie den Äußernsmodus, nachdrücklich' lexikalisieren. Da die Verben des jubeln-Paradigmas und die des klagen-Paradigmas sich nicht nur hinsichtlich der propositionalen Einstellung und der Sprecherabsicht (der negationsinvolvierenden Merkmale), sondern auch in weiteren Aspekten ihrer Bedeutung unterscheiden, sind sie keine Antonyme im engeren Sinn (vgl. Tab. 15). 


\begin{tabular}{|l|l|l|}
\hline \multicolumn{1}{|c|}{$\begin{array}{c}\text { Ausstattung des } \\
\text { Rekurssituationstyps }\end{array}$} & \multicolumn{1}{c|}{$\begin{array}{c}\text { Verben } \\
\text { jubeln, jubilieren, froblocken, } \\
\text { jauchzen, zujubeln }\end{array}$} & $\begin{array}{c}\text { klagen, wehklagen, beklagen, } \\
\text { sich beklagen, sich beschweren, } \\
\text { bedauern, jammern, lamentie- } \\
\text { ren, vorklagen, vorjammern, } \\
\text { vorheulen }\end{array}$ \\
\hline \hline Propositionaler Gehalt (P) & Mitteilungsgehalt: P & Mitteilungsgehalt: P \\
\hline Geschehenstyp (P) & unbestimmt & unbestimmt \\
\hline Zeitbezug (P) & vergangen & vergangen \\
\hline Rollenbezug (P) & unbestimmt & unbestimmt \\
\hline Einstellung von S zu P & S empfindet: Freude wegen P & S empfindet: Leid wegen P \\
\hline Sprecherabsicht & $\begin{array}{l}\text { S will: H erkennt: S empfin- } \\
\text { det: Freude wegen P }\end{array}$ & $\begin{array}{l}\text { S will: H erkennt: S empfin- } \\
\text { det: Leid wegen P }\end{array}$ \\
\hline Vorannahmen von S & P ist der Fall & P ist der Fall \\
\hline Äußernsmodus & nachdrücklich & - \\
\hline
\end{tabular}

Tab. 15: Ausstattung der Rekurssituationstypen JUBELN und KI.AGEN

jubeln jubilieren frohlocken jauchzen zujubeln/schimpfen schelten fluchen verfluchen
wettern geifern giften protestieren

Wenn Emotionen wie Freude und Ärger als Gegensätze aufgefasst werden, können die Verben des jubeln-Paradigmas und die des schimpfen-Paradigmas als Antonyme betrachtet werden: Mit Verben wie jubeln ist die propositionale Einstellung ,S empfindet: Freude wegen P' und die Sprecherabsicht ,S will: $H$ erkennt: $S$ empfindet: Freude wegen P' lexikalisiert; Verben wie schimpfen lexikalisieren die propositionale Einstellung ,S empfindet: Ärger wegen P' und die Sprecherabsicht ,S will: H erkennt: $S$ empfindet: Ärger wegen P'. Zum jubeln-Paradigma gehören auch die Verben jubilieren, frohlocken, jauchzen und zujubeln. Das schimpfen-Paradigma enthält auch die Verben schimpfen, schelten, fluchen, verfluchen, wettern, geifern, giften und protestieren.

Die Verben des jubeln-Paradigmas unterscheiden sich aber auch darin von denen des schimpfenParadigmas, dass sie den Äußernsmodus, nachdrücklich“ lexikalisieren. Da die Verben des jubelnParadigmas und die des schimpfen-Paradigmas sich nicht nur hinsichtlich der propositionalen Einstellung und der Sprecherabsicht (der negationsinvolvierenden Merkmale), sondern auch in weiteren Aspekten ihrer Bedeutung unterscheiden, sind sie keine Antonyme im engeren Sinn (vgl. Tab. 16). 


\begin{tabular}{|c|c|c|}
\hline \multirow{2}{*}{$\begin{array}{c}\text { Ausstattung des } \\
\text { Rekurssituationstyps }\end{array}$} & \multicolumn{2}{|c|}{ Verben } \\
\hline & $\begin{array}{l}\text { jubeln, jubilieren, frohlocken, } \\
\text { jauchzen, zujubeln }\end{array}$ & $\begin{array}{c}\text { schimpfen, schelten, fluchen, } \\
\text { verfluchen, wettern, geifern, gif- } \\
\text { ten, protestieren }\end{array}$ \\
\hline Propositionaler Gehalt (P) & Mitteilungsgehalt: $\mathrm{P}$ & Mitteilungsgehalt: $\mathrm{P}$ \\
\hline Geschehenstyp (P) & unbestimmt & unbestimmt \\
\hline Zeitbezug (P) & vergangen & vergangen \\
\hline Rollenbezug (P) & unbestimmt & unbestimmt \\
\hline Einstellung von $S$ zu $P$ & S empfindet: Freude wegen $P$ & S empfinder: Ärger wegen $P$ \\
\hline Sprecherabsicht & $\begin{array}{l}S \text { will: } H \text { erkennt: } S \text { empfin- } \\
\text { det: Freude wegen } P\end{array}$ & $\begin{array}{l}S \text { will: } H \text { erkennt: } S \text { empfin- } \\
\text { det: Ärger wegen } P\end{array}$ \\
\hline Vorannahmen von S & $P$ ist der Fall & $P$ ist der Fall \\
\hline Äußernsmodus & nachdrücklich & - \\
\hline
\end{tabular}

Tab. 16: Ausstattung der Rekurssituationstypen JUBELN und SCHIMPFEN

\subsubsection{Fälle von Antonymie im engeren Sinn}

(1) Verben, die die propositionale Einstellung ,S findet: P gut' bzw. „S finder: P schlecht‘ lexikalisieren

loben würdigen honorieren huldigen ehrenltadeln rügen rüffeln kritisieren beanstanden bemängeln monieren missbilligen verurteilen anprangern schelten

Die Verben des loben-Paradigmas und die des tadeln-Paradigmas sind Antonyme, weil sie gegensätzliche propositionale Einstellungen und gegensätzliche Sprecherabsichten lexikalisieren: mit Verben wie loben ist die propositionale Einstellung ,S findet: P gut und die Sprecherabsicht ,S will: $\mathrm{H}$ erkennt: $S$ finder: $P$ gut' lexikalisiert; Verben wie tadeln lexikalisieren die propositionale Einstellung ,S findet: P schlecht' und die Sprecherabsicht ,S will: H erkennt: S findet: P schlecht'. Zum loben-Paradigma gehören auch die Verben würdigen, honorieren, huldigen und ehren; das tadeln-Paradigma enthält auch die Verben rügen, rüffeln, kritisieren, beanstanden, bemängeln, monieren, missbilligen, verurteilen, anprangern und schelten.

Da die Verben des loben-Paradigmas und die des tadeln-Paradigmas sich nur hinsichtlich der mit ihnen lexikalisierten propositionalen Einstellung und der Sprecherabsicht (der negationsinvolvierenden Merkmale) unterscheiden, können sie als Antonyme im engeren Sinn betrachtet werden (vgl. Tab. 17). 


\begin{tabular}{|c|c|c|}
\hline \multirow{2}{*}{$\begin{array}{l}\text { Ausstattung des } \\
\text { Rekurssituationstyps }\end{array}$} & \multicolumn{2}{|c|}{ Verben } \\
\hline & $\begin{array}{l}\text { loben, würdigen, honorieren, } \\
\text { huldigen, ehren }\end{array}$ & $\begin{array}{l}\text { tadeln, rügen, rüffeln, kritisie- } \\
\text { ren, beanstanden, bemängeln, } \\
\text { monieren, missbilligen, ver- } \\
\text { urteilen, anprangern, schelten }\end{array}$ \\
\hline Propositionaler Gehalt (P) & Mitteilungsgehalt: P & Mitteilungsgehalt: P \\
\hline Geschehenstyp (P) & Handlung & Handlung \\
\hline Zeitbezug (P) & vergangen & vergangen \\
\hline Rollenbezug (P) & Hörer oder Dritte & Hörer oder Dritte \\
\hline Einstellung von $\mathrm{S}$ zu P & S findet: $\mathrm{P}$ gut & S findet: P schlecht \\
\hline Sprecherabsicht & $\begin{array}{l}\text { S will: } H \text { erkennt: } S \text { findet: } P \\
\text { gut }\end{array}$ & $\begin{array}{l}\text { S will: } H \text { erkennt: } S \text { findet: } P \\
\text { schlecht }\end{array}$ \\
\hline Vorannahmen von $S$ & $P$ ist der Fall & $P$ ist der Fall \\
\hline
\end{tabular}

Tab. 17: Ausstattung der Rekurssituationstypen LOBEN und TADELN

$\mathrm{Da}$ Verben wie loben und solche wie tadeln die propositionale Einstellung , S findet: P gut' bzw. ,S findet: P schlecht' lexikalisieren, kann die Antonymie von loben und tadeln auf die Antonymie von gut und schlecht zurückgeführt werden, die beide skalare Ausdrücke sind. Fraglich ist allerdings, ob die Skalarität von gut und schlecht sich auch auf Verben wie loben und tadeln übertragen lässt. Wenn loben und tadeln keine skalaren Ausdrücke sind, ist unklar, ob sie als konträre Antonyme gelten können. $S$ lobt $H$ und $S$ tadelt $H$ können nicht beide zugleich zutreffen: Ein Sprecher, der jemanden lobt, tadelt diese Person nicht und vice versa $(\mathrm{a} \rightarrow \neg \mathrm{b}$ und $\mathrm{b} \rightarrow \neg \mathrm{a})$. $S$ lobt $H$ und $S$ tadelt $H$ können nur dann beide zugleich nicht zutreffen, wenn ein Sprecher entweder gar nichts tur oder eine völlig andere Handlung vollzieht. Diese Fälle können allerdings nicht mehr der Dimension der sprachlichen Kundgabe einer Bewertung zugeordnet werden. Im Unterschied zu $S$ lobt $H$ und $S$ tadelt $H$ können Äußerungen mit typischen Beispielen konträrer Antonyme wie $X$ ist gut und $X$ ist schlecht beide zugleich nicht zutreffen, ohne dass dies zu einem Dimensionswechsel führt: wenn etwas weder gut noch schlecht ist, kann es dem mittleren Bereich der Skala "gutschlecht" und damit der Dimension „Bewertung“ zugeordnet werden.

Die Verben des loben-Paradigmas und die des tadeln-Paradigmas sind aber auch keine komplementären Antonyme: Während $S$ lobt $H$ und $S$ tadelt $H$ nicht beide zugleich zutreffen können, können $S$ lobt $H$ nicht und $S$ tadelt $H$ nicht schon beide zugleich zutreffen: ein Sprecher kann etwas weder loben noch tadeln, indem er entweder gar nichts macht oder eine völlig andere Handlung vollzieht.

(2) Verben, die die propositionale Einstellung ,S empfindet: Freude wegen P' bzw.

,S empfinder: Ärger wegen P' lexikalisieren

jubeln jubilieren froblocken jauchzen zujubelnlpoltern

Wenn Emotionen wie Freude und Ärger als Gegensätze aufgefasst werden, können die Verben des jubeln-Paradigmas und poltern als Antonyme betrachtet werden: Mit Verben wie jubeln ist die 
propositionale Einstellung ,S empfindet: Freude wegen $P^{\prime}$ und die Sprecherabsicht ,S will: $\mathrm{H}$ erkennt: $S$ empfindet: Freude wegen $P^{\prime}$ lexikalisiert; poltern lexikalisiert die propositionale Einstellung ,S empfindet: Ärger wegen P' und die Sprecherabsicht ,S will: H erkennt: S empfinder: Ärger wegen P'. Zum jubeln-Paradigma gehören auch die Verben jubilieren, froblocken, jauchzen und zujubeln. Das poltern-Paradigma enthält nur das Verb poltern.

$\mathrm{Da}$ die Verben des jubeln-Paradigmas und die des poltern-Paradigmas sich nur hinsichtlich der mit ihnen lexikalisierten propositionalen Einstellung und der Sprecherabsicht (der negationsinvolvierenden Merkmale) unterscheiden, können sie als Antonyme im engeren Sinn betrachtet werden (vgl. Tab. 18).

\begin{tabular}{|l|l|l|}
\hline \multicolumn{1}{c|}{$\begin{array}{c}\text { Ausstattung des } \\
\text { Rekurssituationstyps }\end{array}$} & $\begin{array}{c}\text { jubeln, jubilieren, frohlocken, } \\
\text { jauchzen, zujubeln }\end{array}$ & \multicolumn{1}{c|}{ poltern } \\
\cline { 2 - 3 } & Mitteilungsgehalt: P & Mitteilungsgehalt: $\mathrm{P}$ \\
\hline \hline Propositionaler Gehalt (P) & unbestimmt & unbestimmt \\
\hline Geschehenstyp (P) & vergangen & vergangen \\
\hline Zeitbezug (P) & unbestimmt & unbestimmt \\
\hline Rollenbezug (P) & S empfindet: Freude wegen P & S empfindet: Ärger wegen P \\
\hline Einstellung von S zu P & $\begin{array}{l}\text { S will: H erkennt: S empfin- } \\
\text { det: Freude wegen P }\end{array}$ & $\begin{array}{l}\text { S will: H erkennt: S empfin- } \\
\text { det: Ärger wegen P }\end{array}$ \\
\hline Sprecherabsicht & P ist der Fall & P ist der Fall \\
\hline Vorannahmen von S & nachdrücklich & nachdrücklich \\
\hline Äußernsmodus & & \\
\hline
\end{tabular}

Tab. 18: Ausstattung der Rekurssituationstypen JUBELN und POLTERN

Unklar ist, welcher Typ von antonymischer Beziehung zwischen den Verben des jubeln-Paradigmas und poltern besteht. Obwohl das Haben einer Empfindung wie Freude oder Ärger eine graduierbare mentale Einstellung ist, ist fraglich, ob sich die Skalarität dieser Einstellung auch auf ihre sprachliche Kundgabe übertragen lässt. Unter der Annahme, dass dies nicht der Fall ist, sind Verben wie jubeln und poltern keine skalaren Ausdrücke. Die Möglichkeit ihrer Einordnung als konträre Antonyme ist damit fraglich. Die Einordnung von Verbpaaren wie jubeln-poltern ist auch deswegen problematisch, weil Verben wie jubeln und poltern nicht zwei Bereiche ein und derselben Skala, sondern vielmehr je einen Teilbereich unterschiedlicher Kontinua (des Freudebzw. des Ärger-Kontinuums) bezeichnen.

Verbpaare wie jubeln-poltern sind aber auch keine komplementären Antonyme. Während $S$ jubelt und $S$ poltert nicht beide zugleich zutreffen können, können $S$ jubelt nicht und $S$ poltert nicht schon beide zugleich zutreffen: Ein Sprecher kann weder jubeln noch poltern, indem er gar nichts tut oder eine völlig andere Handlung vollzieht. 


\section{Fazit}

Als Antonymie im engeren Sinn wurde eine Relation des Bedeutungsgegensatzes zwischen zwei lexikalischen Einheiten L(a) und L(b) definiert, die sich nur im Hinblick auf die negationsinvolvierenden Merkmale ihrer Bedeutung unterscheiden. Nach dieser engeren Auffassung von Antonymie können nur die folgenden Paare von Sprechaktverben als Gegensatzbezeichnungen gelten:

1. innerhalb des Paradigmas der Repräsentative

Paare von Verben wie zustimmen-abstreiten

Diese Verben lexikalisieren die folgenden negationsinvolvierenden Merkmale:

\begin{tabular}{|l|l|l|}
\hline \multirow{2}{*}{ Verben } & \multicolumn{2}{|c|}{ Merkmale } \\
\cline { 2 - 3 } & \multicolumn{1}{|c|}{ Propositionale Einstellung } & \multicolumn{1}{|c|}{ Sprecherabsicht } \\
\hline \hline $\begin{array}{l}\text { zustimmen, beipflichten, bestä- } \\
\text { tigen }\end{array}$ & S hält für wahr: $\mathrm{P}$ & $\begin{array}{l}\text { S will: } \mathrm{H} \text { erkennt: } \mathrm{S} \text { hält für } \\
\text { wahr: } \mathrm{P}\end{array}$ \\
\hline $\begin{array}{l}\text { abstreiten, bestreiten, verneinen, } \\
\text { leugnen }\end{array}$ & S hält für wahr: -,P & $\begin{array}{l}\text { S will: } \mathrm{H} \text { erkennt: } \mathrm{S} \text { hält für } \\
\text { wahr: } \rightarrow \mathrm{P}\end{array}$ \\
\hline
\end{tabular}

Tab. 19: zustimmen-abstreiten: negationsinvolvierende Merkmale

2. innerhalb des Paradigmas der Direktive

Keine

3. innerhalb des Paradigmas der Kommissive

Keine

4. innerhalb des Paradigmas der Expressive

Paare von Verben wie loben-tadeln

Diese Verben lexikalisieren die folgenden negationsinvolvierenden Merkmale:

\begin{tabular}{|l|l|l|}
\hline \multirow{2}{*}{ Verben } & \multicolumn{2}{|c|}{ Merkmale } \\
\cline { 2 - 3 } & \multicolumn{1}{|c|}{ Propositionale Einstellung } & \multicolumn{1}{|c|}{ Sprecherabsicht } \\
\hline \hline $\begin{array}{l}\text { loben, würdigen, honorieren, } \\
\text { huldigen, ehren }\end{array}$ & S findet: P gut & $\begin{array}{l}\text { S will: H erkennt: S findet: P } \\
\text { gut }\end{array}$ \\
\hline $\begin{array}{l}\text { tadeln, rügen, rüffeln, kritisie- } \\
\text { ren, beanstanden, bemängeln, } \\
\begin{array}{l}\text { monieren, missbilligen, ver- } \\
\text { urteilen, anprangern, schelten }\end{array}\end{array}$ & S findet: P schlecht & $\begin{array}{l}\text { S will: H erkennt: S findet: } \mathrm{P} \\
\text { schlecht }\end{array}$ \\
\hline
\end{tabular}

Tab. 20: loben-tadeln: negationsinvolvierende Merkmale 
Paare von Verben wie jubeln-poltern

Diese Verben lexikalisieren die folgenden negationsinvolvierenden Merkmale:

\begin{tabular}{|l|l|l|}
\hline \multirow{2}{*}{ Verben } & \multicolumn{2}{|c|}{ Merkmale } \\
\cline { 2 - 3 } & \multicolumn{1}{|c|}{ Propositionale Einstellung } & \multicolumn{1}{c|}{ Sprecherabsicht } \\
\hline \hline $\begin{array}{l}\text { jubeln, jubilieren, frohlocken, } \\
\text { jauchzen, zujubeln }\end{array}$ & S empfindet: Freude wegen P & $\begin{array}{l}\text { S will: H erkennt: S empfin- } \\
\text { det: Freude wegen P }\end{array}$ \\
\hline poltern & S empfindet: Ärger wegen P & $\begin{array}{l}\text { S will: H erkennt: S empfin- } \\
\text { det: Ärger wegen P }\end{array}$ \\
\hline
\end{tabular}

Tab. 21: jubeln-schimpfen: negationsinvolvierende Merkmale

Keines dieser Verbpaare erfüllt die Bedingungen für komplementäre oder konträre Antonymie. Zwei lexikalische Ausdrücke L(a) und L(b) müssen zwei Bedingungen erfüllen, um als komplementäre bzw. konträre Antonyme gelten zu können. Die erste dieser Bedingungen gilt sowohl für komplementäre als auch für konträre Antonymie:

Bedingung I (komplementäre und konträre Antonymie):

a und $b$ können nicht beide zugleich zutreffen

Diese Bedingung wird von allen Verbpaaren, die hier als Antonyme im engeren Sinn aufgeführt sind, erfüllt. Zusätzlich gilt für komplementäre Antonymie die Bedingung IIa und für konträre Antonymie die Bedingung IIb:

\section{Bedingung IIa (komplementäre Antonymie):}

a und $b$ können nicht beide zugleich nicht zutreffen

Diese Bedingung wird von keinem der hier aufgeführten Paare antonymischer Sprechaktverben erfüllt: Es ist möglich, dass ein Sprecher weder die durch das eine noch die durch das andere Verb bezeichnete sprachliche Handlung vollzieht, indem er gar nichts tut oder eine völlig andere Handlung vollzieht.

\section{Bedingung IIb (konträre Antonymie):}

$\mathrm{a}$ und $\mathrm{b}$ können beide zugleich nicht zutreffen

Die hier aufgeführten Paare antonymischer Sprechaktverben erfüllen diese Bedingung in einer anderen Weise als die typischen Beispiele konträrer Antonyme wie etwa gut-schlecht, groß-klein und glücklich-unglücklich: Es ist möglich, dass ein Sprecher weder die durch das eine noch die durch das andere Verb bezeichnete sprachliche Handlung vollzieht, indem er gar nichts tur oder eine völlig andere Handlung vollzieht. Solche Fälle können allerdings nicht mehr der durch die beiden Verben bezeichneten Dimension zugeordnet werden. Äußerungen mit typischen Beispielen konträrer Antonyme können beide zugleich nicht zutreffen, ohne dass dies zu einem Dimensionswechsel führt. Wenn etwas beispielsweise weder groß noch klein ist, kann es dem mittleren Bereich der Skala der möglichen Größen und damit der Dimension „Größe“ zugeordnet werden. Diese Möglichkeit besteht bei Paaren antonymischer Sprechaktverben nicht, weil sie keine skalaren Ausdrücke sind. 